\title{
The Continuous, Desingularized Newton Method for Meromorphic Functions
}

\author{
H. TH. JONGEN, P. JONKER, and F. TWILT \\ Twente University of Technology, Department of Applied Mathematics, P.O. Box 217, 7500 AE \\ Enschede, The Netherlands
}

(Received: 17 October 1986)

\begin{abstract}
For any (nonconstant) meromorphic function, we present a real analytic dynamical system, which may be interpreted as an infinitesimal version of Newton's method for finding its zeros. A fairly complete description of the local and global features of the phase portrait of such a system is obtained (especially, if the function behaves not too bizarre at infinity). Moreover, in the case of rational functions, structural stability aspects are studied. For a generic class of rational functions, we give a complete graph-theoretical characterization, resp. classification, of these systems. Finally, we present some results on the asymptotic behaviour of meromorphic functions.
\end{abstract}

AMS subject classifications (1980). 05C05, 05C30, 30D30, 30D35, 34C35, 58F09.

Key words. Newton method, meromorphic function, dynamical system, phase-portrait, plane (sphere) graph, asymptotic value.

\section{Introduction and Motivation}

Let $\mathbb{C}$ be the complex plane and $f$ be a complex function on $\mathbb{C}$. The function $f$ is called entire if it is (complex) analytic everywhere on $\mathbb{C} ; f$ is called meromorphic if all its singularities (i.e., points of $\mathbb{C}$ where $f$ fails to be analytic) are poles.

In this paper, $f$ is always a nonconstant meromorphic function. By $N(f)(P(f))$ we denote the set of all zeros (poles) for $f$. If $f^{\prime}$ stands for the usual derivative of $f$, we call $C(f):=N\left(f^{\prime}\right) \backslash N(f)$ the critical set of $f$; its elements are called critical points for $f$. If $f\left(z_{0}\right)=f^{\prime}\left(z_{0}\right)=0$ or if $z_{0} \in P(f)$, the singularity for $f / f^{\prime}$ can be removed by defining $f / f^{\prime}\left(z_{0}\right)=0$. In general, a meromorphic function, which has a removable singularity at $z_{0}$ will be interpreted as to be analytic at $z_{0}$ (a same convention holds w.r.t. removable singularities of real analytic mappings).

This paper is concerned with the study of dynamical systems of the form

$$
\frac{\mathrm{d} z(t)}{\mathrm{d} t}=-\frac{f(z(t))}{f^{\prime}(z(t))}
$$

Obviously, the system (1.1) is not defined at critical points for $f$. However, it is possible to 'desingularize' (1.1) by introducing a real analytic dynamical system which is defined on the whole $\mathbb{C}$ such that, on $\mathbb{C l} C(f)$, its maximal trajectories coincide with those of (1.1). In some special cases, it is even possible to extend this system to a real analytic system on the 2 -sphere $S^{2}$, viewed as a one-point 
compactification of the complex plane. Once we have obtained such a desingularization and extension, we can use the whole machinery of the theory on (real analytic) dynamical systems on the plane and sphere (as well as the theory on functions of a complex variable).

In order to motivate the work presented here, we indicate how it fits into a more general framework and give some references to the literature.

Let us consider differential equations of the form

$$
\frac{\mathrm{d} x(t)}{\mathrm{d} t}=-\mathrm{D} F^{-1}(x(t)) \cdot F(x(t))
$$

where $F \in C^{2}\left(\mathbf{R}^{n}, \mathbf{R}^{n}\right)$, the space of twice continuously differentiable mappings from $\mathbf{R}^{n}$ to $\mathbf{R}^{n}$, and $\mathrm{D} F(x)$ stands for the matrix of partial derivatives at $x$. Apparently, the r.h.s. of (1.2) is not defined on the set $C:=\left\{x \in \mathbb{R}^{n} \mid \operatorname{det} \mathrm{DF}(x)=\right.$ $0\}$; so we have to assume that $x(t) \notin C$.

If we write down Euler's approximation [16] to system (1.2), then we find:

$$
x_{k+1}=x_{k}-h_{k} \cdot \mathrm{D} F^{-1}\left(x_{k}\right) \cdot F\left(x_{k}\right), \quad k=0,1,2, \ldots,
$$

where $h_{k}$ are positive reals which may be suitably chosen. This is the well-known (relaxed) Newton-Raphson iteration method for finding zeros for $F$. Therefore, the system (1.2) may be considered as a 'continuous' Newton method.

The fact that (1.2) is not defined on the set $C$ causes a lot of trouble, both from the theoretical and computational points of view; in fact, $C$ may be very irregular and near $C$ the r.h.s. of (1.2) may blow up. Following Branin [3], Gomulka [7], Hirsch and Smale [14], and Smale [31], we may overcome this difficulty by considering the system

$$
\frac{\mathrm{d} x(t)}{\mathrm{d} t}=-\tilde{\mathrm{D}} F(x(t)) \cdot F(x(t))
$$

where $\tilde{\mathrm{D}} F(x)$ stands for the well-known adjoint matrix of $\mathrm{D} F(x)$ and, hence

$$
\tilde{\mathrm{D}} F(x) \cdot \mathrm{D} F(x)=\operatorname{det} \mathrm{D} F(x) \cdot I_{n},
$$

$I_{n}$ being the $n \times n$ unit matrix, cf. [7]. System (1.4) is well-defined on the whole $\mathbf{R}^{n}$. Outside the set $C$ we have $\tilde{\mathrm{D}} F(x) \cdot F(x)=\operatorname{det} \mathrm{D} F(x) \cdot \mathrm{D} F^{-1}(x) \cdot F(x)$. Thus, on $\mathbf{R}^{n} \backslash C$ the phase portraits of (1.2) and (1.4) are 'equal' (eventually - depending on the sign of det $\mathrm{D} F(x)$ - up to orientation). That is why the system (1.4) will be referred to as to a 'continuous, desingularized' Newton method.

As it is clear from the literature on (chaotic) discrete dynamic systems, in connection with Newton's method (cf. [17,27]), the global convergence behaviour of (1.3) can be very wild and unsurveyable, even in the case of simple mappings $F$. In order to get a better insight into the global aspects of Newton's iteration method (1.3), it is not, therefore, unreasonable to treat - as a first step a continuous, desingularized version (such as (1.4)). A key fact is the following 
observation: multiplying both sides of (1.4) by $D F(x(t))$ yields, in view of (1.5) and applying the chain rule:

$$
\frac{\mathrm{d}}{\mathrm{d} t} F(x(t))=-\operatorname{det} \mathrm{D} F(x(t)) \cdot F(x(t)) .
$$

Hence, $F$ maps trajectories of (1.4) to half-lines in $\mathbf{R}^{n}$. This fact makes it possible to cast the investigation of the phase portrait of (1.4) in a differentialgeometrical setting, namely the study of the inverse images under $F$ of half rays in $\mathbf{R}^{\boldsymbol{n}}$. On the basis of these ideas, Smale obtained interesting results on the global convergence behaviour of the discrete and the continuous (desingularized) Newton method. However, Smale's results deal with special aspects and, moreover, require rather strong conditions on $F$ and DF. (For example, in [14] an algorithm is proposed for finding the zeros for mappings $F$ which must be, among other conditions, real analytic and proper [8].) Especially, in the general case of mappings $F \in C^{2}\left(\mathbf{R}^{n}, \mathbf{R}^{n}\right)$ with $n>2$, there is no hope of a powerful theory which holds under mild conditions and by means of which it is possible to describe the phase portrait of (1.4) in detail. Nevertheless, the approach given above maintains a certain intrinsic value, since it clarifies the geometrical background of various algorithms for finding zeros for $F$ (see, e.g., [5]).

So, in order to obtain a more or less general theory, we have to restrict ourselves to certain subsets of $C^{2}\left(\mathbf{R}^{n}, \mathbf{R}^{n}\right), n>2$, or to the case $n=2$. As a matter of fact, the results obtained by Hirsch and Smale [14] and Smale [31] do hold for a generic subset of $C^{2}\left(\mathbf{R}^{n}, \mathbf{R}^{n}\right)$. In our paper [18], we studied systems (1.4) for 'gradient mappings' (i.e., mappings $F$ of the form grad $g$, where $g$ is a smooth function from $\mathbf{R}^{n}$ to $\left.\mathbf{R}\right)$ and introduced a generic subset $E(n)$ of the space of all smooth functions on $\mathbf{R}^{n}$, such that: (1) For $g \in E(n)$, the set $C$ is a closed Whitney stratified subset of $\mathbf{R}^{n}$ of dimension $\leqslant(n-1)$; (2) the equilibrium states of (1.4) which are contained in $C$ (the so-called extraneous singularities) constitute a Whitney stratified set of a dimension $\leqslant(n-2)$; (3) global convergence properties in the same spirit as those obtained by Smale in [31] do hold; (4) in the case $n=2$ a fairly complete description fo the phase portrait of (1.4) is given, including a statement on structural stability.

Now, let us return to the case of entire functions $f$ (considered as $C^{2}$-mappings $\mathbf{R}^{2} \rightarrow \mathbf{R}^{2}$, the components of which are related by the Cauchy-Riemann equations). Then, (1.2) takes the form of (1.1), whereas for (1.4) we find

$$
\frac{\mathrm{d} z(t)}{\mathrm{d} t}=-\overline{f^{\prime}}(z(t)) \cdot f(z(t)) .
$$

Here, $\overline{f^{\prime}}(z(t))$ stands for the complex conjugate of $f^{\prime}(z(t))$. Note that, in this case we have $C=N\left(f^{\prime}\right)$, so if $f$ has multiple zeros, then $C(f)$ is a strict subset of C.

The polynomial case, where everything is relatively simple (e.g., $N(f)$ and 
$C(f)$ are finite sets; $P(f)=\emptyset)$ is treated by Braess [2] and by Hirsch and Smale [14], and Smale [32]. Note that the only proper, entire functions are the polynomials.

In our papers $[19,20]$ we extended most of the results obtained in [2] to the case of meromorphic functions. Note that a strictly meromorphic function (i.e., $f$ has at least one pole) is not contained in $C^{2}\left(\mathbf{R}^{2}, \mathbf{R}^{2}\right)$. Therefore, another 'desingularization' step is needed, similar to the change-over from (1.1) to (1.6).

This study is organized as follows: Section 2 treats local and global properties of the phase portrait of (1.1) in the general case of meromorphic functions, including the desingularization result. For some special classes of functions, an extension of (1.1) to the sphere is presented. Sections 3 and 4 deal with the case of rational functions. In Section 3, we select a generic subclass of the set of all rational functions, such that the phase-portraits of the corresponding systems (1.1) behave regularly under small perturbations of the coefficients (of these rational functions). We call these functions nondegenerate and the corresponding systems (1.1) structurally stable. With any nondegenerate function, with degree (denominator) < degree (numerator), a connected plane graph is associated which fulfils a simple combinatorial condition. Conversely, any connected planegraph with this property represents a system (1.1) corresponding with a nondegenerate rational function with degree (denominator) $<$ degree (numerator). This is shown in Section 4. Using this result, in Section 5 we obtain a complete classification of all structurally stable systems (1.1). Finally, in Section 6, we present (as an application) some results on the asymptotic behaviour of entire and meromorphic functions.

This present work is close to our papers $[19,20]$. As a matter of fact, Sections 2, 3 (partially), and 6 merely constitute a survey of results obtained in [19], the proofs being very roughly sketched or deleted. Finally, we refer to Twilt [33] where most of the results reported here are to be found in full detail.

\section{The General Case}

In this section we summarize the local and global properties of dynamical systems of the form (1.1). For proofs and more details we refer to [19].

The right-hand side $\left(-(f(z)) /\left(f^{\prime}(z)\right)\right)$ of $(1.1)$, considered as a complex analytic vector field on $\mathbb{C l} C(f)$, is denoted by $\mathcal{N}(f)$; it is referred to as to "the Newton flow for $f^{\prime}$. Throughout this paper, a trajectory of $\mathcal{N}(f)$ through $z_{0}(\notin C(f))$ is always interpreted as maximal and will be denoted by $\gamma\left(z_{0}\right)$.

Apparently, the only equilibrium states for $\mathcal{N}(f)$ are the zeros and poles for $f$, i.e., if $z_{0} \in N(f) \cup P(f)$ then $\gamma\left(z_{0}\right)=\left\{z_{0}\right\}$.

In the case where $z_{0} \notin C(f) \cup N(f) \cup P(f)$, the trajectory $\gamma\left(z_{0}\right)$ is called regular and is given by the solution

$$
\left.z(t), z_{0}=z(0), \quad t \in\right] a, b[, \quad \text { eventually } a=-\infty, b=+\infty .
$$


By direct integration, we find

$$
\left.f(z(t))=\mathrm{e}^{-t} f\left(z_{0}\right), \quad t \in\right] a, b[.
$$

Thus, on $\gamma\left(z_{0}\right)$ we have $\arg f(z)=$ constant $\left(=\arg f\left(z_{0}\right)\right)$. Hence, $\gamma\left(z_{0}\right)$ is contained in the inverse image under $f$ of the line $\arg w=\arg f\left(z_{0}\right)$; cf. Section 1 .

From this and from the elementary properties of (multifold) conformal mappings, one immediately concludes that the local phase portrait of $\mathcal{N}(f)$ around a point (say $z_{0}$ ) is of one of the four types (cf. [23]) shown in Figure 1.

In Figure $1 \mathrm{~b}$ (Figure 1c), let $l=$ multiplicity of $z_{0}$ as a zero (pole) for $f, l \geqslant 1$. Adopting the terminology of Hartman [12], $z_{0}$ is a stable (unstable) proper node for $\mathcal{N}(f)$, i.e., for every $\Theta \in[0,2 \pi[$, a unique trajectory $\gamma$ exists such that if $z$ tends to $z_{0}$ along $\gamma$, then $\arg \left(z-z_{0}\right)$ tends to $\Theta$. The angle $\phi$ in Figure $1 \mathrm{~b}$ (Figure 1c) equals

$$
\frac{1}{l} \arg \frac{f\left(z_{1}\right)}{f\left(z_{2}\right)}\left(\frac{1}{l} \arg \frac{\left(f\left(z_{2}\right)\right)}{\left(f\left(z_{1}\right)\right)}\right) .
$$

In Figure $1 \mathrm{~d}$, let $k=$ multiplicity of $z_{0}$ as a zero for $f^{\prime}, k \geqslant 1$. Then, the angle between two subsequent trajectories, one of which tends to $z_{0}$, the other leaves from $z_{0}$, equals $\pi /(k+1)$. In this case, we call $z_{0}$ a critical point of order $k$ for $f$ (or $k$-fold saddlepoint for $\mathcal{N}(f)$ ). In the figure, we obviously have $k=2$.

Now, we focus our attention to the global aspects of the phase-portrait of $\mathcal{N}(f)$. The limiting set of a (regular) trajectory $\gamma\left(z_{0}\right)$ is extremely simple, as is pointed out in the following lemma.

LEMMA 2.1 (The limiting sets of trajectories).

(1) Either $\lim _{t \uparrow b} z(t)=\infty$ or $\lim _{t \uparrow b} z(t)=z_{*} \in \mathbb{C}$; in the latter case we have $z_{*} \in N(f)$ if $b=\infty$ and $z_{*} \in C(f)$ if $b<\infty$.

(2) Either $\lim _{t \downarrow a} z(t)=\infty$ or $\lim _{t \downarrow a} z(t)=z_{*} \in \mathbb{C}$; in the latter case we have $z_{*} \in P(f)$ if $a=-\infty$ and $z_{*} \in C(f)$ if $a>-\infty$.

We emphasize that, although Lemma 2.1 is in the same spirit as Bendixon's theorem on limiting sets of trajectories in the plane, it requires an independent proof (cf. [19]). However, in the (special) case where $\mathcal{N}(f)$ can be extended to the

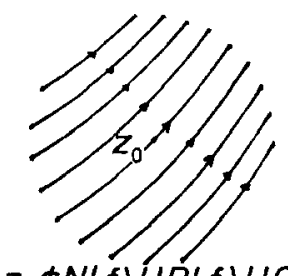

$z_{0} \notin N(f) \cup P(f) \cup C(f)$

(a)

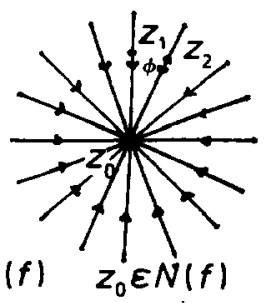

(b)
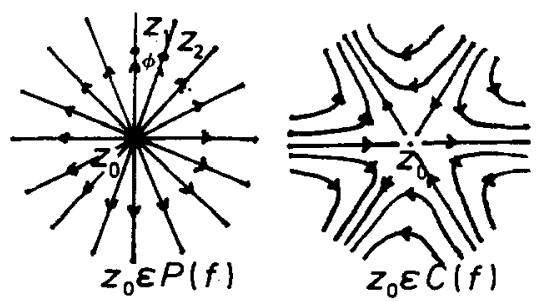

(c) (k-fold saddle -

(d) point)

Fig. 1. 
whole Riemannian sphere (in the sense of the forthcoming Lemma 2.2), Lemma 2.1 is a straightforward consequence of the Poincaré-Bendixon-Schwartz theorem for dynamical systems on the sphere and the fact that in view of relation (2.1) periodic trajectories and a so-called path polygon with trajectories spiralling to it, cannot occur (see also [22]).

Intuitively speaking, the basin $B\left(z_{*}\right)$ of a zero (pole) $z_{*}$ for $f$ is the set of points 'moving' to (from) $z_{*}$ along a trajectory of $\mathcal{N}(f)$. More precisely,

$$
B\left(z_{*}\right):=\left\{z_{*}\right\} \cup\left\{z_{0} \in \mathbb{C} \mid \lim z(t)=z_{*} ; z_{0}=z(0)\right\},
$$

where the limit is taken $t \rightarrow+\infty$ if $z_{*} \in N(f)$, respectively $t \rightarrow-\infty$ if $z_{*} \in P(f)$.

Let $\partial B\left(z_{*}\right)$ be the boundary of $B\left(z_{*}\right)$. Then, we have

$$
\partial B\left(z_{*}\right)=\emptyset \quad \text { iff } f \text { is of the form } f(z)=\alpha\left(z-z_{*}\right)^{n},
$$

where $\alpha \in \mathbb{C}$ and $n$ is a positive (negative) natural number if $z_{*}$ is a zero (pole).

The crucial point in the proof (cf. [19]) is to reject the possibility that $-\partial B\left(z_{*}\right)$ being nonempty - for $z_{*} \in \mathcal{N}(f)$ (resp. $\in P(f)$ ), the function $f$ (resp. $1 / f$ ) is transcendental entire and possesses only one zero (pole) and no critical points. This follows by application of the Casorati-Weierstrass theorem and Relation (2.1).

In the case where $\partial B\left(z_{*}\right)$ is nonempty, it is the union of the (topological) closures of regular trajectories of $\mathcal{N}(f)$. This follows from a careful analysis of what happens to points in a sufficiently small neighbourhood of $\partial B\left(z_{*}\right)$, which is essentially based on the continuous dependence of solutions of $(1.1)$ on the initial conditions (cf. [12]) and on relation (2.1) again.

In the case where $B\left(z_{*}\right)$ is bounded (thus $\partial B\left(z_{*}\right) \neq \emptyset$ ), on the boundary of $B\left(z_{*}\right)$ there lies at least one pole (zero) if $z *$ is a zero (pole) for $f$. Note that this result implies that the basin of a zero for an entire function is unbounded (compare also Braess [2], who treated the polynomial case).

If we try to exploit the theory on two-dimensional dynamical systems at full strength, we encounter the problem that $\mathcal{N}(f)$ is not defined on the whole $\mathbb{C}$. We overcome this difficulty by means of the following 'desingularization' lemma:

LEMMA 2.2 (Desingularization Lemma). For each nonconstant meromorphic function $f$, a real analytic vector field - say $\overline{\mathcal{N}}(f)$ - exists, defined on the whole $\mathbb{C}$, with the properties:

(1) Trajectories for $\mathcal{N}(f)$ are also trajectories for $\overline{\mathcal{N}}(f)$

(2) A critical point for $f$ is an equilibrium state for $\overline{\mathcal{N}}(f)$

(3) $\overline{\mathcal{N}}(f)=-\overline{\mathcal{N}}(1 / f)$

Here, we merely give the explicit formula for $\overline{\mathcal{N}}(f)$, namely

$$
\overline{\mathcal{N}}(f)=-\left(1+|f(z)|^{4}\right)^{-1} \cdot \bar{f}^{\prime}(z) \cdot f(z)
$$

(compare also the change-over from (1.1) to (1.6) in Section 1). 
For special choices of the function $f$, it is even possible to extend $\overline{\mathcal{N}}(f)-$ by means of the transformation $w=1 / z$ - to a real analytic vector field on the 2 -sphere $S^{2}$. Here, we treat two choices:

(1) The set $(\mathscr{R})$ of all nonconstant rational functions $f$ (i.e., $f=p_{n} / q_{m}$, with $p_{n}$ (resp. $q_{m}$ ) polynomials of degree $n(m)$ which are relatively prime) and

(2) The set $(\mathscr{E})$ of all transcendental entire functions $f$ of order $l(<\infty)$ with finitely many zeros (i.e., $f=u_{r} \cdot \exp v_{l}$; with $u_{r}$ (resp. $v_{l}$ ) polynomials of degree $r$ (resp. $l$ ); note that $l \geqslant 1$ since $f$ is transcendental).

LEMMA 2.3 (Extension to $S^{2}$ ). For each $f \in \mathscr{R} \cup \mathscr{E}$, there exists a real analytic vector field $\overline{\overline{\mathcal{N}}}(f)$, defined on the whole $S^{2}$, such that on the $z$-chart of $S^{2}$ (i.e., $S^{2} \backslash\{z=\infty\}$ ) the phase portrait of $\overline{\overline{\mathcal{N}}}(f)$ equals the phase portrait of $\overline{\mathcal{N}}(f)$ (up to a stereographic projection). Moreover, for $f \in \mathscr{R}$, we have $\overline{\overline{\mathcal{N}}}(f)=-\overline{\overline{\mathcal{N}}}(1 / f)$.

For later use we give the explicit expressions for $\overline{\overline{\mathcal{N}}}(f)$ w.r.t. the $z$-chart and the $w$-chart $\left(\overline{\overline{\mathcal{N}}}_{z}(f)\right.$ resp. $\left.\overline{\overline{\mathcal{N}}}_{w}(f)\right)$ :

If $f \in \mathscr{R}, n \neq m$

$$
\begin{aligned}
& \overline{\overline{\mathcal{N}}}_{z}(f)=-\left(1+|z|^{2}\right)^{|n-m|+1} \cdot\left(1+|f(z)|^{4}\right)^{-1} \cdot \overline{f^{\prime}}(z) \cdot f(z), \\
& \overline{\overline{\mathcal{N}}}_{w}(f)=-\left(1+|w|^{2}\right)^{|n-m|+1} \cdot|w|^{-2|n-m|+2} \cdot\left(1+\left|f\left(\frac{1}{w}\right)\right|^{4}\right)^{-1} \cdot \frac{\mathrm{d}}{\mathrm{d} w} f\left(\frac{1}{w}\right) \cdot f\left(\frac{1}{w}\right) .
\end{aligned}
$$

If $f \in \mathscr{R}, \boldsymbol{n}=\boldsymbol{m}$

$$
\begin{aligned}
& \overline{\overline{\mathcal{N}}}_{z}(f)=-\left(1+|z|^{2}\right)^{2} \cdot\left(1+|f(z)|^{4}\right)^{-1} \cdot \overline{f^{\prime}}(z) \cdot f(z), \\
& \overline{\overline{\mathcal{N}}}_{w}(f)=-\left(1+|w|^{2}\right)^{2} \cdot\left(1+\left|f\left(\frac{1}{w}\right)\right|^{4}\right)^{-1} \cdot \frac{\mathrm{d}}{\mathrm{d} w} f\left(\frac{1}{w}\right) \cdot f\left(\frac{1}{w}\right) .
\end{aligned}
$$

If $f \in \mathscr{E}$

$$
\begin{aligned}
& \overline{\overline{\mathcal{N}}}_{z}(f)=-\left(1+|z|^{2}\right)^{-l-r+1}\left(\overline{u_{r}^{\prime}}(z)+\overline{v_{l}^{\prime}}(z) \cdot \overline{u_{r}}(z)\right) \cdot u_{r}(z) \\
& \overline{\overline{\mathcal{N}}}_{w}(f)=-\left(1+|w|^{2}\right)^{-l-r+1} \cdot|w|^{2 l+2 r+2} \cdot\left[\overline{\frac{\mathrm{d} u_{r}}{\mathrm{~d} w}\left(\frac{1}{w}\right)}+\frac{\overline{\mathrm{d} v_{l}}}{\mathrm{~d} w}\left(\frac{1}{w}\right) \cdot \bar{u}_{r}\left(\frac{1}{w}\right)\right] \cdot u_{r}\left(\frac{1}{w}\right)
\end{aligned}
$$

In all these cases, the pair $\left(\overline{\overline{\mathcal{N}}}_{z}(f), \overline{\overline{\mathcal{N}}}_{w}(f)\right)$ constitutes a real analytic vector field on $S^{2}$. For a verification of this statement (especially of the fact that the singularity of $\overline{\overline{\mathcal{N}}}_{w}(f)$ at $w=0$ is removable), we refer to [19].

Note that, in general, an extension of $\overline{\mathcal{N}}(f)$, with $f$ meromorphic, to a real analytic $\overline{\overline{\mathcal{N}}}(f)$ on $S^{2}$ (as demonstrated in the preceding lemma) is not possible. This follows from the existence of meromorphic functions, whose finite zeros do accumulate at $z=\infty$ (e.g., $\tan z$ ).

As in the case of the finite equilibrium states for $\overline{\mathcal{N}}(f)$ (cf. Figure 1; Lemma 2.1) for those functions $f$ for which Lemma 2.3 is valid, we can also give a complete description of the local phase portrait of $\overline{\overline{\mathcal{N}}}(f)$ around $z=\infty$. To this 
aim; we introduce for $f \in \mathscr{R}, f=p_{n} / q_{m}$, the following integers: $s=$ total number of critical points for $f$, each counted a number of times equal to its order, and if $n=m$, we define $k=2 n$-degree $\left(p_{n}^{\prime} \cdot q_{n}-p_{n} \cdot q_{n}^{\prime}\right)-2$.

LEMMA 2.4. (The local phase-portrait around $z=\infty$ ). (a) For $f \in \mathscr{R}, f=p_{n} / q_{m}$, we have

- If $n<m($ resp. $n>m): z=\infty$ is an (un-)stable nondegenerate node for $\overline{\overline{\mathcal{N}}}(f)$

- If $n=m: z=\infty$ is a regular point for $\overline{\overline{\mathcal{N}}}(f)$ (in the case $k=0$ ), or $z=\infty$ is a $k$-fold saddlepoint for $\overline{\overline{\mathcal{N}}}(f)$ (in the case $k \geqslant 1$ ).

Moreover, $k=\# N(f)+\# P(f)-s-2$, where \# denotes cardinality.

(b) For $f \in \mathscr{E}, f=u_{r} \exp v_{l}$, we have:

- $z=\infty$ is an isolated, degenerate equilibrium state for $\overline{\overline{\mathcal{N}}}(f)$ with - apart from the (eventual) parabolic sectors - exactly $2 l$ elliptic sectors, denoted by $\epsilon_{j}$, $j=1, \ldots, 2 l$, and no hyperbolic sectors.

- for each $j$, there exist two rays, say $L_{j, 1}, L_{j, 2}$ emanating from $w=0$, determining the angle $\pi / l$ and with the property that each trajectory in $\epsilon_{j}$ emanates from $w=0$ tangent to $L_{j, 1}$ and trends to $w=0$ tangent to $L_{j, 2}$.

The proof of Lemma 2.4 follows from a straightforward (but quite technical) analysis of the expressions for $\overline{\overline{\mathcal{N}}}(f)$ proceeding Lemma 2.3 , an application of the theorem of 'Poincaré-Hopf on dynamical systems on $\mathrm{S}^{2}$ with only isolated equilibrium states' (cf. [25]) and 'Bendixon's formula for the Poincaré index of an isolated, nonrotational equilibrium state' (cf. [12]).

REMARK 2.1. The fundamental theorem of algebra follows directly - by considering $f(z)=\left(p_{n}(z)\right) / z^{n}$ - from the second statement of Lemma 2.4a and the facts that $k \geqslant 0$ and $s \geqslant 0$.

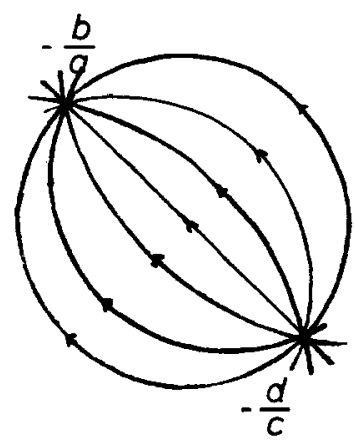

$\mathcal{N}(f) ; a c \neq 0$

(a)

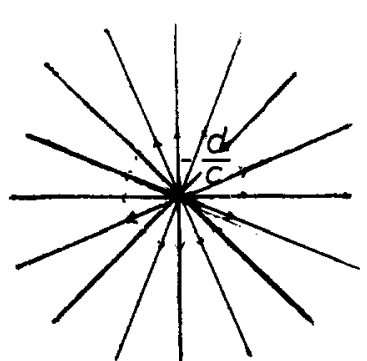

$\mathcal{N}(f) ; a=0$

(b)

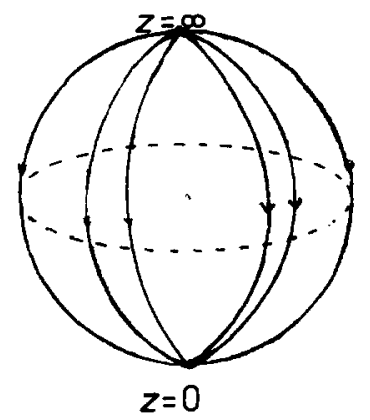

$\overline{\mathcal{N}}(f) ; b=c=0$

(c)

Fig. 2. 


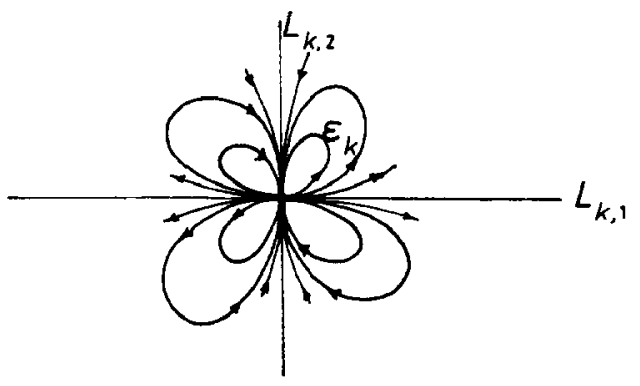

Fig. 3.

For more results on the structure of (the boundary of) a basin of a node for $\overline{\bar{N}}(f)$, with $f \in \mathscr{R} \cup \mathscr{E}$, we refer to [19], and to Section 3 .

EXAMPLE 2.1. Let

$$
f(z)=\left(\frac{a z+b}{c z+d}\right)^{n}
$$

with $a d \neq b c$ and $n=1,2,3 \ldots$ In the case where $a c \neq 0$ we have:

$$
N(f)=\left\{-\frac{b}{a}\right\}, P(f)=\left\{-\frac{d}{c}\right\}, C(f)=\emptyset
$$

and $z=\infty$ is a regular point of $\overline{\overline{\mathcal{N}}}(f)$. If $a=0$, then $z=\infty$ is the only zero for $f$; $P(f)=\{-(d / c)\} ; C(f)=\emptyset$. If $c=0$, then $z=\infty$ is the only pole for $f ; N(f)=$ $\{-(b / a)\} ; C(f)=\emptyset$.

The system $\mathcal{N}(f)$ (or $\overline{\overline{\mathcal{N}}}(f)$ ) is called 'north-south flow' (cf. Figure 2). Note that the cases $a=0$, resp. $c=0$, provide the only examples of Newton flows with the property that the boundary of the basin of one of its nodes is empty.

EXAMPLE 2.2. Let $f(z)=z \exp (z+8)^{2}$. Obviously, $f \in \mathscr{E}$; order $f=2$. One easily verifies that the intervals into which the real axis is subdivided by the zero $(z=0)$ and the two simple critical points $-4-\frac{1}{2} \sqrt{62},-4+\frac{1}{2} \sqrt{62}$ constitute trajectories for $\mathcal{N}(f)$. Hence, it follows that the system $\overline{\overline{\mathcal{N}}}(f)$ has exactly four elliptic sectors at $z=\infty$ (cf. Lemma 2.4b and Figure 3).

\section{The Case of Rational Functions and Structural Stability Aspects}

In this section, we restrict ourselves to the (nonconstant) functions $f$ in $\mathscr{R}$. Such functions can be extended (in the usual way) to meromorphic functions on the sphere $S^{2}$; the set of all these extensions will also be denoted by $\mathscr{R}$. The point $z=\infty$ is called (infinite) zero, pole, critical point for $f$ if $w=0$ is, respectively, a zero, pole, critical point for the function $f(1 / w)$, where $w=1 / z$.

As in Section 2, a function $f \in \mathscr{R}$ will always be represented by $p_{n} / q_{m}$, where 
$p_{n}, q_{m}$ are relatively prime polynomials of degree $n, m$. Note that in this representation, $p_{n}$ and $q_{m}$ are unique up to a common scalar. By $\mathscr{R}_{+}, \mathscr{R}_{0}, \mathscr{R}_{-}$we mean the set of functions $f=p_{n} / q_{m}$ with, respectively, $n>m, n=m, n<m$.

The aim of this section is to select a 'generic' subset ( $\mathscr{R})$ of functions $f$, such that small perturbations of the coefficients of $f$ (not changing the degree of numerator, denominator) do not alter the qualitative features of the phase portraits of the resulting Newton systems ('structural stability'). To make this more precise, we have to introduce a topology $(\tau)$ on $\mathscr{R}$ and an equivalence relation $(\sim)$ on the set of all rational Newton flows (i.e., systems of the form $\overline{\overline{\mathcal{N}}}(f)$, with $f \in \mathscr{R}$ ).

We conclude this section by associating with each $f\left(=p_{n} / q_{m}\right)$ in $\tilde{\mathscr{R}}$ for which $n>m$, a plane-graph $\bar{G}(f)$, which fulfils the so-called Cycle Property.

\subsection{THE TOPOLOGY $\tau$}

The set $\mathscr{R}$ may be endowed with a topology $(\tau)$ which is natural in the following sense: Let $f \in \mathscr{R}$ be represented by $p_{n} / q_{m}$. Given $\epsilon>0$ sufficiently small, then there exists a $\tau$-neighbourhood $\Omega$ of $f$ such that for each $g \in \Omega$, the function $g$ can be represented by $\tilde{p}_{n} / \tilde{q}_{m}$ such that the coefficients of $\tilde{p}_{n}, \tilde{q}_{m}$ are in $\epsilon$ neighbourhoods of the corresponding coefficients of $p_{n}, q_{m}$. For a precise definition of $\tau$, we refer to [19].

Let $\chi\left(S^{2}\right)$ stand for the set of all $C^{1}$-differentiable dynamical systems on $S^{2}$ endowed with the $C^{1}$-topology $(c)$, cf. [1]. In view of Lemma 2.3, the map $T$ : $\mathscr{R} \rightarrow \chi\left(S^{2}\right): T(f)=\overline{\overline{\mathcal{N}}}(f)$ is well-defined. We proved in [19] the following useful result which 'connects' the topologies $\tau$ and $c$.

LEMMA 3.1. $T$ is $(\tau, c)$-continuous.

\subsection{EQUIVALENCE OF (RATIONAL) NEWTON FLOWS AND STRUCTURAL STABILITY ASPECTS}

The systems $\Sigma$ and $\Sigma^{\prime}$ in $\chi\left(S^{2}\right)$ are called topologically equivalent $\left(\Sigma \sim \Sigma^{\prime}\right)$ if a homeomorphism from $S^{2}$ to $S^{2}$ exists which maps the trajectories of $\Sigma$ onto those of $\Sigma^{\prime}$ and preserves the orientation of the trajectories. (Informally, this means $\Sigma$ and $\Sigma^{\prime}$ have the same qualitative behaviour.)

The system $\Sigma$ is called structurally stable if a $c$-neighbourhood $\mathcal{O}$ of $\Sigma$ exists such that for each $\Sigma^{\prime} \in \mathcal{O}$ we have $\Sigma \sim \Sigma^{\prime}$.

The following characterization of structural stability is due to de Baggis and Peixoto.

A system $\Sigma \in \chi\left(S^{2}\right)$ which does not exhibit periodic trajectories is structurally stable iff the following two properties hold: (1) Each equilibrium-state for $\Sigma$ is hyperbolic, cf. [13] p. 187, and thus nondegenerate; (2) no two saddlepoints of $\Sigma$ are 'connected' by a trajectory. 
In the special case of rational Newton flows on the sphere, we introduce a concept of structural stability which takes into account small perturbations of the coefficients of the underlying functions:

The system $\overline{\overline{\mathcal{N}}}(f)$ is called structurally stable (as a rational Newton flow) if a $\tau$-neighbourhood $\Omega$ of $f$ exists such that for each $g \in \Omega$ we have $\overline{\overline{\mathcal{N}}}(f) \sim \overline{\overline{\mathcal{N}}}(\underline{g})$.

So, we have introduced two concepts of structural stability for systems $\overline{\mathcal{N}}(f)$. However, it turns out that both concepts coincide. In fact, from Lemma 3.1 it follows that if $\overline{\overline{\mathcal{N}}}(f)$ is structurally stable (as an element of $\chi\left(S^{2}\right)$ ), then it is also structurally stable as a rational Newton flow. The converse is also true. This follows from the forthcoming Theorem 3.1(ii), Lemma 3.2 and the de BaggisPeixoto characterization of structural stability mentioned above. (Note that in view of Relation (2.1), the system $\overline{\bar{N}}(f)$ has no periodic trajectories.)

Under a global boundary condition, structural stability may also be defined for systems in the plane. In the following context, it plays an important role in Section 4.

For $R>0$ we define $D_{R}\left(C_{R}\right)$ as the disc (circle) in $\mathbb{C}$ given by $|z| \leqslant R(|z|=R)$. We define $\chi(R)$ as the set of restrictions to $D_{R}$ of $C^{1}$-vector fields on a neighbourhood of $D_{R}$ for which $C_{R}$ is a global boundary (i.e., the vector fields are transversal to $\left.C_{R}\right)$. The space $\chi(R)$ is endowed with the $C^{1}$-topology. With respect to $\chi(R)$, we may introduce structural stability (including the above characterization results) in the same way as in the case of $\chi\left(S^{2}\right)$, cf. [1].

Moreover, if $X \in \chi(R)$ is structurally stable and $\epsilon>0$ arbitrary, then for $Y \in \chi(R)$, 'sufficiently' $C^{1}$-close to $X$, an $\epsilon$-homeomorphism $\Psi: D_{R} \rightarrow D_{R}$ exists (i.e., $\psi$ is a homeomorphism and moreover, $\|\psi(x)-x\|<\epsilon$, all $x \in D_{R}$ ) such that $\psi$ maps the trajectories of $X$ onto those of $Y$ (respecting their orientations), cf. [29]. Therefore, $X$ is also called $\epsilon$-structurally stable.

\subsection{THE SET (色) OF NONDEGENERATE RATIONAL FUNCTIONS}

The function $f \in \mathscr{R}$ is called nondegenerate if

(1) All finite zeros and poles are simple.

(2) All critical points for $f$ - eventually including $z=\infty$ - are simple (as zeros for $f^{\prime}$ ).

(3) No two critical points for $f$ are 'connected' by a trajectory of $\overline{\bar{N}}(f)$.

The subset of $\mathscr{R}$ consisting of all nondegenerate functions is denoted by $\tilde{R}$. Moreover, $\tilde{\mathscr{R}}_{+}:=\tilde{\mathscr{R}} \cap \mathscr{R}_{+}$, whereas $\tilde{\mathscr{R}}_{0}$ and $\tilde{\mathscr{R}}_{-}$are defined in a similar way.

By direct inspection of the expressions of the vector fields $\overline{\overline{\mathcal{N}}}(f)$, cf. Lemmata 2.3 and $2.4 a$, we find

LEMMA 3.2. If $f \in \tilde{\mathscr{R}}$, then, all equilibrium-states of $\overline{\overline{\mathcal{N}}}(f)$ are hyperbolic.

Using this result, the de Baggis-Peixoto characterization of structural stability as 
mentioned above, as well as the very properties of the topology $\tau$, we come to the main result of this section (cf. [19]).

Theorem 3.1 (Genericity and characterization of rational Newton flows).

(i) $\tilde{\mathscr{R}}$ is $\tau$-open and $\tau$-dense in $\mathscr{R}$.

(ii) $\overline{\overline{\mathcal{N}}}(f)$ is structurally stable iff $f \in \tilde{\mathscr{R}}$.

REMARK 3.1. There is a physical interpretation of this result: one easily sees that (1.1) yields the differential equation for the streamlines of a steady stream with complex potential $-\log f(z)$, cf. [23]. So, we may expect that in the case where, extended to the sphere, the stream has only finitely many 'sources' and 'sinks' (this corresponds to $f \in \mathscr{R}$ ), in general, the phase portrait of $\overline{\overline{\mathcal{N}}}(f)$ behaves extremely regular w.r.t. small perturbations of the coefficients of $f$.

\subsection{THE GRAPH $\bar{G}(f)$ AND THE CYCLE PROPERTY}

Let us consider a function $f \in \tilde{R}_{+}$, i.e., $f\left(=p_{n} / q_{m}\right)$ is nondegenerate and $n>m$. In view of the nondegeneracy of $f$, there are exactly $n$ zeros and $m$ finite poles for $f$ (all simple) denoted by resp. $\omega_{i}, i=1, \ldots, n$ and $\alpha_{j}, j=1, \ldots, m$. For $z=\infty, f$ has a pole (and thus $\overline{\overline{\mathcal{N}}}(f)$ has an unstable node at $z=\infty$ ).

The Poincare indices (cf. [8]) of the (un-)stable nodes resp. the saddlepoints of $\overline{\overline{\mathcal{N}}}(f)$ equal +1 resp. -1 . Application of the Poincaré-Hopf Theorem ([25]) to $\overline{\overline{\mathcal{N}}}(f)$, yields

$$
n+(m+1)-\# C(f)=2 .
$$

Hence, $C(f)=\emptyset$ iff $n=1$; in that case $\overline{\overline{\mathcal{N}}}(f)$ is a 'north-south flow', cf. Example 2.1 .

If $n>1$, the critical points for $f$ are denoted by $\sigma_{k}, k=1, \ldots s$, where $s=\# C(f)$. The (un-)stable manifold at $\sigma_{k}$ of $\overline{\mathcal{N}}(f)$ (cf. Lemma 2.2) is defined as the union of $\left\{\sigma_{k}\right\}$ and both (un-)stable separatrices at $\sigma_{k}$.

Now, we are in a position to associate with $f$ a plane graph $\vec{G}(f)$ (i.e., a realization of an abstract graph, say $G(f)$, in the plane).

DEFINITION 3.1. For $f \in \tilde{\mathscr{R}}_{+}$, the plane-graph $\bar{G}(f)$ is defined as follows:

- The vertices are the zeros for $f$.

- The edges are the (topological) closures of the unstable manifolds at the saddlepoints of $\overline{\overline{\mathcal{N}}}(f)$.

Note that this definition makes sense in view of Lemma 2.1 and because $f \in \tilde{R}_{+}$. From the nondegeneracy of $f$ and from Relation (2.1), it follows that each unstable manifold at a saddlepoint connects two different zeros (of $f$ ); this means that $\bar{G}(f)$ does not admit edges joining a vertex to itself ('loops'). Multiple edges (i.e., two vertices joined by more than one edge) may occur; see the forthcoming Examples 3.2 and 3.3 . 
If we delete from the plane all edges and vertices of the graph $\bar{G}(f)$, the connected components of the resulting set are called the regions of $\bar{G}(f)$. These regions are just the basins of the poles for $f$ (see Section 2) and will be denoted by $a_{0}, \ldots a_{m}$. Here, $a_{0}$ stands for the unbounded region (the basin of the infinite pole $z=\infty$ ) and $a_{j}$ is the region containing $\alpha_{j}, j=1, \ldots, m$. The number of the bounded regions (cyclomatic number) is denoted by $\mu(\bar{G}(f))$; apparently, we have $\mu(\bar{G}(f))=m$. The well-known Euler 'polyhedron-formula' (cf. [6]), for plane graphs:

$$
\text { \# (regions) }-\#(\text { edges })+\#(\text { vertices })-\#(\text { components })=1
$$

yields in our case

$$
(m+1)-s+n-\#(\text { components of } \bar{G}(f))=1 .
$$

Together with (3.1), this yields \# (components of $\bar{G}(f))=1$, i.e., $\bar{G}(f)$ is a connected graph.

The vertices of $\bar{G}(f)$ are the stable proper nodes of $\mathcal{N}(f)$. Consequently, the angle between two different edges of $\bar{G}(f)$ at a common vertex is well-defined and does not vanish.

Let $C$ be a cycle of $\bar{G}(f)$, i.e., $C$ is a closed Jordan curve which is built up from an alternating sequence of vertices and edges. We define the numbers $n_{C}$, $r_{C}$ and $l_{C}$ as follows

$$
\begin{aligned}
& n_{C}:=\text { number of } \bar{G}(f) \text {-vertices in } \operatorname{Int}(C)(=\# N(f) \cap \operatorname{Int}(C)), \\
& r_{C}:=\text { number of } \bar{G}(f) \text {-regions in } \operatorname{Int}(C)(=\# P(f) \cap \operatorname{Int}(C)),
\end{aligned}
$$

where $\operatorname{Int}(C)$ stands for the interior of $C$.

$$
l_{C}:=\text { number of } \bar{G}(f) \text {-edges on } C \text { (= number of } \bar{G}(f) \text {-vertices on } C \text { ). }
$$

The vertices of $C$ are denoted by $\omega_{C(l)}, l=1, \ldots, l_{C}$. The angles between two consecutive edges of $C$ spanning a sector of $\operatorname{Int}(C)$ at $\omega_{C(l)}$ are given by $2 \pi \phi_{C(l)}$, where $0<\phi_{C(l)}<1, l=1, \ldots, l_{C}$ (see Figure 4 ).

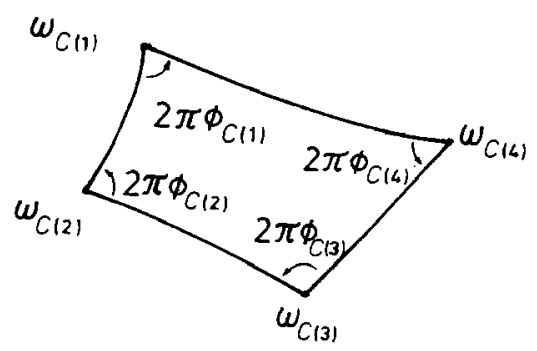

Fig. 4. 
The Cauchy principal value (cf. [23]) of

$$
\oint_{C} \frac{f^{\prime}(z)}{f(z)} \mathrm{d} z
$$

is well-defined, and since the zeros and finite poles for $f$ are simple, we find for it

$$
\oint_{C} \frac{f^{\prime}(z)}{f(z)} \mathrm{d} z=2 \pi i\left[\left(\sum_{l=1}^{l_{C}} \phi_{C(l)}\right)+n_{C}-r_{C}\right]
$$

Since $C$ is built up by trajectories of the system

$$
\frac{\mathrm{d} z}{\mathrm{~d} t}=-\frac{f(z(t))}{f^{\prime}(z(t))} \quad(\text { cf. }(1.1))
$$

one easily derives that the 1.h.s. of $(*)$ is a real value and consequently:

$$
\sum_{l=1}^{l_{C}} \phi_{C(l)}=r_{C}-n_{C} \text {. }
$$

In view of the inequalities $0<\phi_{C(l)}<1$ for each $\phi_{C(l)}$, we have proved the following lemma.

LEMMA 3.3 (Cycle Property). Let $f \in \tilde{R}_{+}$, then for each cycle $C$ in $\bar{G}(f)$ we have

$$
n_{C}<r_{C}<n_{C}+l_{C}
$$

Note that, although in $\bar{G}(f)$ multiple edges may occur, from the above Cycle Property we see that it is impossible for two $\bar{G}(f)$-vertices to be connected by more than two edges.

EXAMPLE 3.1. In the case where $f(\epsilon \tilde{\mathscr{R}})$ is a polynomial, $\bar{G}(f)$ is a connected plane graph without cycles (a so-called tree). This easily follows from the properties of $\bar{G}(f)$ which were derived above.

Let us consider $p(z)=\left(z-z_{1}\right)\left(z-z_{2}\right)\left(z-z_{3}\right)$, with $z_{1}, z_{2}, z_{3}$ noncollinear. By elementary means, one proves (cf. [2,19]) that $p \in \tilde{\mathscr{R}}$ iff the triangle with $z_{1}, z_{2}$, $z_{3}$ as its vertices has exactly one longest side. From this it follows that the polynomial $p$, given by

$$
p(z)=z(z-1)(z-a), \quad a=\frac{1}{10}+\frac{4}{10} i
$$

is nondegenerate. In Figure 5a some of the trajectories of the phase-portrait of $\mathcal{N}(p)$ are depicted; the graph $\bar{G}(p)$ is indicated by the solid line in Figure $5 b$.

EXAMPLE 3.2. Consider the system

$$
\mathcal{N}\left(f_{n}\right) ; f_{n}(z)=\frac{z^{n}-1}{7}, \quad n \geqslant 2 .
$$




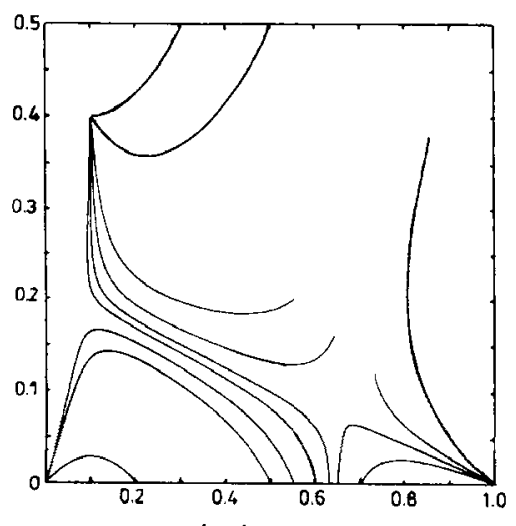

(a)

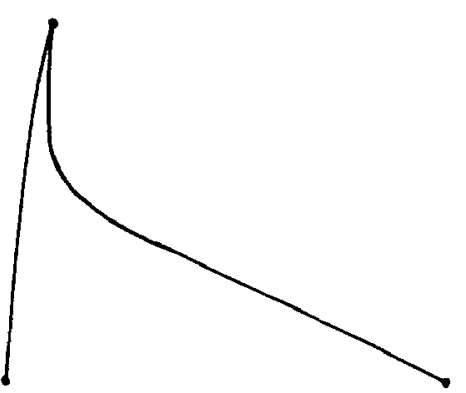

(b)

Fig. 5.

Obviously,

$$
\begin{aligned}
& N\left(f_{n}\right)=\left\{\exp \left(\frac{2 k \pi i}{n}\right) \mid k=0, \ldots, n-1\right\} ; \quad P\left(f_{n}\right)=\{0\} \\
& C\left(f_{n}\right)=\left\{(n-1)^{-1 / n} \cdot \exp \left[\left(\frac{\pi}{n}+\frac{2 k \pi}{n}\right) i\right] \mid k=0, \ldots, n-1\right\} .
\end{aligned}
$$

One easily verifies that arg $f_{n}\left(\sigma_{i}\right) \neq \arg f_{n}\left(\sigma_{j}\right)$ if $i \neq j$ where $\sigma_{i}, \sigma_{j} \in C\left(f_{n}\right)$ and that all finite poles and all zeros for $f_{n}$ are simple. It follows that $f_{n} \in \tilde{\mathscr{R}}_{+}$and that $\bar{G}\left(f_{n}\right)$ has one cycle. From a symmetry argument one deduces that all vertices of $\bar{G}\left(f_{n}\right)$ are on its cycle.

In Figure $6 \mathrm{a}, \mathrm{b}$ the phase-portraits in the case $n=2$, resp. $n=5$, are indicated (the dotted lines give $\bar{G}\left(f_{n}\right)$ ). Case $n=2$ delivers an example of a graph $\bar{G}(f)$ with multiple edges.

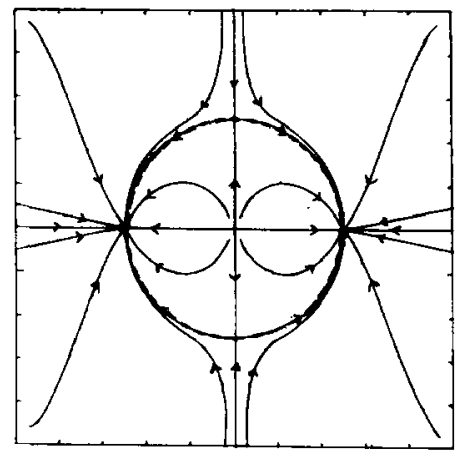

(a)

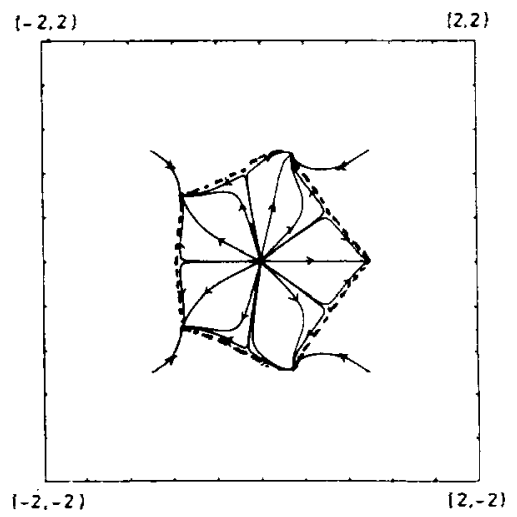

(b)

Fig. 6. 
EXAMPLE 3.3. Consider the function

$$
f(z)=\frac{\left[(z+2)^{2}-1\right]\left[(z-2)^{2}-1\right]}{\left(z^{2}-4\right)}
$$

Obviously,

$$
\begin{aligned}
& N(f)=\{-3,-1,1,3\} ; \quad P(f)=\{-2,2\} ; \\
& C(f)=\{ \pm \sqrt{4+i \sqrt{15}}, \pm \sqrt{4-i \sqrt{15}}, 0\},
\end{aligned}
$$

where $\sqrt{ }$ stands for the usual principal value of the square root.

One easily shows that the positive and negative imaginary axes are (regular) trajectories of $\mathcal{N}(f)$. This is also the case for the intervals into which the real axis is subdivided by the zeros, poles and critical points for $f$. Using a symmetry argument, one finds that the phase-portrait of $\mathcal{N}(f)$ is of the form as depicted in Figure 7a; especially one finds that $f$ is nondegenerate. In Figure $7 \mathrm{~b}$, the graph $\bar{G}(f)$ is depicted.

EXAMPLE 3.4. Let

$$
f(z)=\frac{(z+1)(z+2)}{z} \text {. }
$$

One easily shows that both unstable separatrices at the (simple) critical point $\sqrt{2}$ as well as both stable separatrices at the (simple) critical point $-\sqrt{2}$ lie on the circle with center $z=0$ and radius $\sqrt{2}$. From this, one concludes that $f$ is degenerate. In Figure 8 some of the trajectories of $\mathcal{N}(f)$ are drawn.

REMARK 3.2. The phase portraits in Figures $5 \mathrm{a}, 6 \mathrm{a}, \mathrm{b}$ and 8 are obtained by plotting-out, using a PDP-11 computer, the approximate trajectories of the Newton flows involved, w.r.t. a suitable discretization (cf. (1.3)).

Obviously, the Cycle Property (see Lemma 3.3) may be formulated for any connected plane graph. Two plane graphs $\bar{G}_{1}, \bar{G}_{2}$ are called equivalent $\left(\bar{G}_{1} \sim \bar{G}_{2}\right)$ if a homeomorphism $\phi$ from the plane onto itself exists such that under $\phi$ the edges and vertices of $\bar{G}_{1}$ are mapped onto the edges and vertices of $\bar{G}_{2}$.

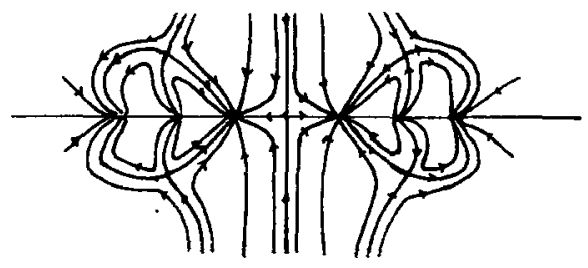

(a)

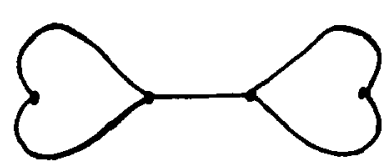

(b)

Fig. 7. 


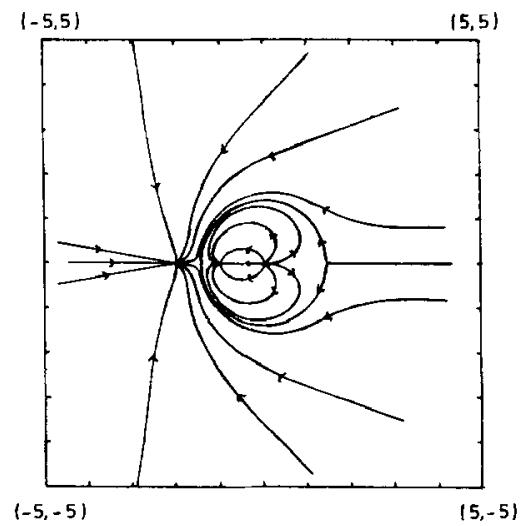

Fig. 8.

Apparently, this induces an equivalence relation on the set of all connected plane-graphs, which respects the Cycle Property.

REMARK 3.3. A necessary condition for the above-introduced equivalence $\bar{G}_{1} \sim \bar{G}_{2}$ is that the underlying (abstract) graphs $G_{1}, G_{2}$ are isomorphic, the isomorphism being such that either at each vertex the cyclic order of the edges incident with this vertex is preserved or is, at each vertex, reversed. One can prove that this condition is also sufficient under the additional claim that the boundaries of the unbounded regions (considered as subgraphs) correspond under the isomorphism (cf. [33]).

REMARK 3.4. In the general case of functions $f \in \mathscr{R}$, one can define a sphere graph $\overline{\bar{H}}(f)$ with as vertices the zeros and critical points for $f$ (eventually including $z=\infty$ ) and as edges the closures of the unstable separatrices of $\overline{\overline{\mathcal{N}}}(f)$ at its saddlepoints. As in the case of $\bar{G}(f)$, one can prove, by using Poincaré's index theorem and Euler's polyhedron formula on the sphere, that $\bar{H}(f)$ is connected (cf. [33]). In the special case where $f \in \tilde{\mathscr{R}}$, we can define the spherical analogue of $\bar{G}(f)$, i.e., a sphere graph $\overline{\bar{G}}(f)$ with as vertices the zeros for $f$ on $S^{2}$ and as edges the closures of the unstable manifolds of $\overline{\overline{\mathcal{N}}}(f)$ at its saddlepoints (cf. [33]). Note that $\overline{\bar{H}}(f), f \in \tilde{R}$, may be obtained from $\overline{\bar{G}}(f)$ by subdividing each edge into two edges. Hence, $\overline{\bar{G}}(f)$ is connected. If $f \in \tilde{\mathscr{R}}_{+}$, then $\overline{\bar{G}}(f)$ is the image of $\bar{G}(f)$ under the stereographic projection from $\mathbb{C}$ onto $S^{2} \backslash\{z=\infty\}$, cf. Lemma 2.3. In view of the Relation $\overline{\overline{\mathcal{N}}}(f)=-\overline{\overline{\mathcal{N}}}(1 / f)$, we have: $\overline{\bar{G}}(1 / f)$ is the geometrical dual of $\overline{\bar{G}}(f)$.

\section{The Representation Theorem}

In this section we introduce a special type of plane graph: the Newton graph. We show that $\bar{G}(f), f \in \tilde{\mathscr{R}}_{+}$, is such a Newton graph (Lemma 4.2). Conversely - and 
this requires a much harder proof - any Newton graph is equivalent to a graph $\bar{G}(f)$, some $f \in \tilde{R}_{+}$(Corollary 4.2).

Let $\bar{G}$ be a connected plane graph with vertices $\omega_{i}, i=1, \ldots, n$, and regions $r_{j}, j=0, \ldots \mu$, where $r_{0}$ stands for the unbounded region. The boundary of $r_{j}$, considered as a subgraph of $\bar{G}$, is denoted by $\partial r_{j}$. By deg $\omega_{i}$ we denote the degree of $\omega_{i}$ (= number of edges, incident with $\omega_{i}$ ).

We assume that the concept of angle between any two edges with a common vertex is well defined. This assumption is reasonable since an arbitrary plane graph is always equivalent to a plane graph with polygons as edges (cf. [6]) and since our ultimate aim is to characterize, up to equivalence, certain rational Newton flows in terms of plane graphs. Moreover, we exclude the case of a 'one-vertex graph', so we assume $n \geqslant 2$.

For each $\omega_{i}$, with deg $\omega_{i}>1$, the embedding of $\bar{G}$ in the plane induces a cyclic anticlockwise order on the edges at $\omega_{i}$. By

$$
2 \pi \omega_{i(k)}, \quad k=1, \ldots, \operatorname{deg} \omega_{i}, 0 \leqslant \omega_{i(k)} \leqslant 1,
$$

we denote the angles at $\omega_{i}$ of two edges which are consecutive with respect to this order (and measured from the first edge to the second one in an anticlockwise sense). If deg $\omega_{i}=1$, then we define $\omega_{i(1)}=1$.

The set

$$
A(\bar{G}):=\left\{\omega_{i(k)} \mid i=1, \ldots, n ; k=1, \ldots, \operatorname{deg} \omega_{i}\right\}
$$

is called the set of angles for $\bar{G}$. We define for each $j, j=1, \ldots \mu$ :

$$
a\left(\partial r_{j}\right):=\left\{\omega_{i(k)} \in A(\bar{G}) \mid \omega_{i} \in \partial r_{j} ; \omega_{i(k)} \text { spans a sector of } r_{j}\right\} .
$$

DEFINITION 4.1 (Newton graph). $\bar{G}$ is called a Newton-graph if

(i) $0<\omega_{i(k)}<1$, for all $i$ with $\operatorname{deg} \omega_{i}>1$ and all $k \in\left\{1, \ldots, \operatorname{deg} \omega_{i}\right\}$,

(ii) $\sum_{a\left(\partial_{i}\right)} \omega_{i(k)}=1$, for $j=1, \ldots, \mu$.

Note that, if $\bar{G}$ is a tree, i.e., $\mu=0$, then condition (ii) is trivially fulfilled. It is easily seen that a Newton graph $\bar{G}$ contains no loops and, moreover, we have $\mu<n$. (In the case of a tree the last assertion is trivial; if $\bar{G}$ is not a tree, it follows by observing that

$$
\sum_{j=1}^{\mu}\left(\sum_{a\left(\partial r_{j}\right)} \omega_{i(k)}\right)=\mu, \sum_{i=1}^{n}\left(\sum_{k=1}^{\operatorname{deg} \omega_{i}} \omega_{i(k)}\right)=n
$$

for all $i \in\{1, \ldots, n\}$ and $\partial r_{0} \neq \emptyset$.)

REMARK 4.1. From a graph-theoretical point of view, the definition of Newton graph is not very satisfactory, since it deals with the concept of angle. However, it is possible to give a purely combinatorial criterion for an arbitrary plane graph $\bar{G}$ to be equivalent to a Newton graph, namely $\bar{G}$ must fulfil the Cycle Property (see Lemma 3.3). One side of the assertion follows directly from Definition 4.1. 
The proof of the other side, see [33], would blow up the size of this paper, and will be published separately (cf. [21]).

Let $\vec{G}$ be a Newton graph with $\mu \geqslant 1$. Then, for each $j \in\{1, \ldots, \mu\}$, the subgraph $\partial r_{j}$ is Eulerian (i.e., a closed eulerian trail $\left(\tau_{j}\right)$ exists: an alternating sequence of vertices and edges beginning and ending with the same vertex and containing all vertices and edges of $\partial r_{j}$, but each edge only once; see Figure 9). We construct such a trail $\tau_{j}$ as follows: choose an arbitrary edge, say $x_{1}$, of $\partial r_{j}$. Then, $x_{1}$ is incident with two different vertices of degree $>1$. This follows from the facts that $x_{1}$ is not a loop, $r_{j}$ is bounded and the very definition of the Newton graph. Only one of these vertices, say $\omega_{1}$, has the property that the angle at $\omega_{1}$ between $x_{1}$ and the $\bar{G}$-edge proceeding $x_{1}$ (w.r.t. the anticlockwise ordering of edges, incident with $\left.\omega_{1}\right)$ belongs to $a\left(\partial r_{j}\right)$. The other vertex in $x_{1}$, say $\omega_{2}$, has the exclusive property that the angle at $\omega_{2}$ between $x_{1}$ and the $\bar{G}$-edge at $\omega_{2}$, say $x_{2}$, preceding $x_{1}$ belongs to $a\left(\partial r_{j}\right)$. Note that the uniqueness is a consequence of Condition (i) in Definition 4.1. Now, we let $x_{2}$ play the role of $x_{1}$ above. We find two (different) $x_{2}$-vertices: $\omega_{2}$ and $\omega_{3}$ (in the role of $\omega_{1}$ resp. $\omega_{2}$ w.r.t. $x_{1}$ ) and an edge $x_{3}$ (in the role of $x_{2}$ ). So, we can go on. The procedure stops if we achieve the edge $x_{1}$ again. The resulting sequence of vertices and edges is an Eulerian trail (in fact here we need Definition 4.1 again). Using the Cycle Property, a more formal construction of $\tau_{j}$ is given in [33], compare Remark 4.1. The uniquely determined angle between two consecutive edges in $\tau_{j}$ at a common vertex which spans a sector of $r_{j}$ is referred to as to 'the angle of $\tau_{j}$ of this consecutive pair at the common vertex'.

Let us consider a function $f \in \tilde{\mathscr{R}}_{+}$which is not a polynomial of degree 1 (thus $\mathcal{N}(f)$ is not a north-south flow, cf. Example 2.1). We recall that $\overline{\overline{\mathcal{N}}}(f)$ is structurally stable. Moreover, $\overline{\overline{\mathcal{N}}}(f)$ has an unstable, nondegenerate proper node at $z=\infty$ and does not exhibit periodic trajectories.

The canonical regions of $\overline{\mathcal{N}}(f)$ are the connected components of the set which is obtained by deleting from $\mathbb{C}$ the (topological) closures of the stable and unstable manifolds at the saddlepoints of $\overline{\mathcal{N}}(f)$.

As a direct consequence of the results of Peixoto $[28,30]$ on structurally stable

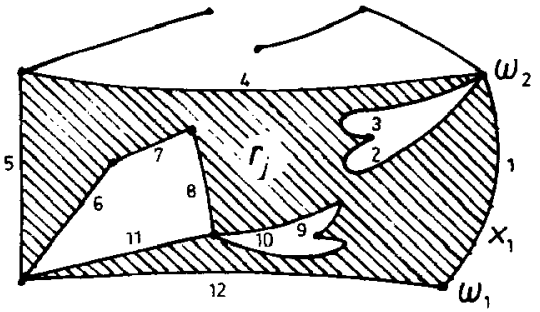

The labels $1, \ldots, 12$ give the orientation of the Euler trail $\tau_{i}$ of $\partial r_{j}$.

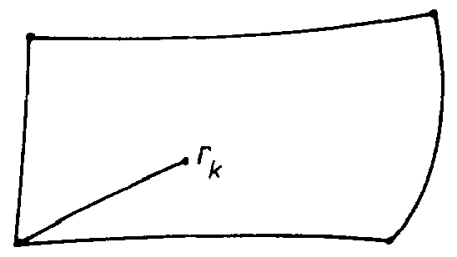

$\partial r_{k}$ is not an Euler trail. 


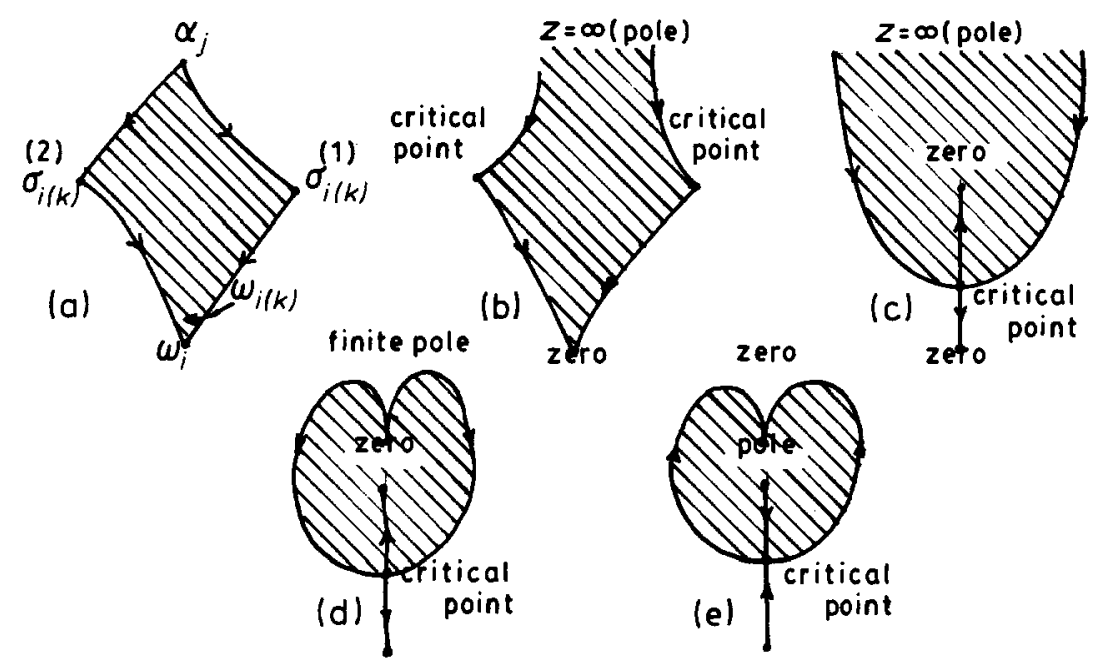

Fig. 10.

systems on $S^{2}$, a canonical region of $\overline{\mathcal{N}}(f)$ is, a priori, one of the types as depicted in Figure 10.

However, since we deal with systems $\overline{\mathcal{N}}(f), f \in \tilde{\mathscr{R}}_{+}$, canonical regions of the type as depicted in Figure 10d,e do not occur. This follows from the facts that

(1) In view of the Relation (2.1), we have on trajectories of $\overline{\mathcal{N}}(f)$ : $\arg$ $f(z)=$ constant.

(2) The zeros and finite poles for $f$ are simple.

(3) The zeros and poles for $f$ provide proper nodes of $\overline{\mathcal{N}}(f)$.

The existence of the other types follow from the examples given in Section 3.

In an obvious way (see Figure 10) each angle $\omega_{i(k)} \in A(\bar{G}(f))$ determines exactly one of the canonical regions of $\overline{\mathcal{N}}(f)$. We denote this canonical region by $A_{i(k)}(f)$ or, if no confusion is possible, by $A_{i(k)}$.

Let $\omega_{i_{0}}$ be a zero for $f(=$ vertex of $\bar{G}(f))$ such that deg $\omega_{i_{0}}>1$ (see Figure $10 \mathrm{a}, \mathrm{b})$. Then there are exactly two critical points for $f$ on the boundary of each $A_{i_{0}(k)}, k=1, \ldots, \operatorname{deg} \omega_{i_{1}}$. For later use we introduce the following notation: if $\omega_{i_{1}(k)}$ is measured (in an anticlockwise sense) from the $\bar{G}(f)$-edge $x_{1}$ to the $\bar{G}(f)$-edge $x_{2}$, then the critical point on $x_{1}\left(x_{2}\right)$ is denoted by $\sigma_{i_{0}(k)}^{(1)}\left(\sigma_{i_{0}(k)}^{(2)}\right)$.

Next, let $\omega_{i}$, be a zero for $f\left(=\right.$ vertex of $\bar{G}(f)$ ) such that deg $\omega_{i_{1}}=1$ (see Figure 10c). Then, there is exactly one critical point for $f$ on the boundary of $A_{i_{1}(1)}$. This critical point is denoted by $\sigma_{i_{1}(1)}$.

LEMMA 4.1. Let $f \in \tilde{\mathscr{R}}_{+}$and $f \neq$ (polynomial of degree 1 ). For each zero $\omega_{i}$ with $\operatorname{deg} \omega_{i}>1$ we have

$$
\omega_{i(k)}=\frac{1}{2 \pi} \arg \frac{f\left(\sigma_{i(k)}^{(2)}\right)}{f\left(\sigma_{i(k)}^{(1)}\right)}
$$


where arg stands for the principal value of the argument function with the cut along the positive real axis.

Proof. The function $f$ is conformal at $\omega_{i}$ (since $\omega_{i}$ is a simple zero for $f$ ). Consequently, $f$ is angle-preserving at $\omega_{i}$. From this, the assertion follows since on trajectories of $\overline{\mathcal{N}}(f)$ we have $\arg f(z)=$ const.

Note that the argument of the quotient in Lemma 4.1 are taken over complex numbers which are not contained in the interval $[0, \infty[$.

COROLLARY 4.1. If $A_{i(k)}$ is a bounded canonical region (see Figure 10a) then the angle at the pole equals the angle at the zero.

Proof. This follows from the previous lemma and the Relation $\overline{\mathcal{N}}(f)=$ $-\overline{\mathcal{N}}(1 / f)$.

LEMMA 4.2. Let $f \in \tilde{R}_{+}$and $f \neq$ (polynomial of deg. 1 ). Then, $\bar{G}(f)$ is a Newton graph.

Proof. The bounded region $r_{j}$ of $\bar{G}(f)$ contains the pole $\alpha_{j}$. All canonical regions of $\overline{\mathcal{N}}(f)$ in $r_{j}$ are of the form as depicted in Figure 10a with $\alpha_{j}$ as a pole. Condition (ii) of Definition 4.1 therefore follows directly from Corollary 4.1; condition (i) of Definition 4.1 is fulfilled since the zeros for $f$ are proper stable nodes of $\mathcal{N}(f)$ (see Section 2).

For $R>0$, we consider again the circle $C_{R}:=\{z|| z \mid=R\}$. Since $z=\infty$ is an unstable proper node for the system $\overline{\overline{\mathcal{N}}}(f)$ (Lemma 2.4) an $R_{0}>0$ exists such that for all $R, R>R_{0}$, we have that $C_{R}$ is global boundary for $\overline{\mathcal{N}}(f)$. This implies that $N(f) \cup C(f) \cup P(f)$ is contained in $\operatorname{Int}\left(C_{R}\right)$.

Let $R>R_{0}$.

For unbounded canonical regions $A_{i(k)}$ (see Figure $10 \mathrm{~b}, \mathrm{c}$ ) the intersection $A_{i(k)} \cap C_{R}$ is a connected open arc, denoted arc $(R)$. The positive angle spanned by this arc is denoted by $2 \pi C_{i(k)}^{R}$.

LEMMA 4.3. Let $A_{i(k)}$ be an unbounded canonical region determined by the $\bar{G}(f)$-angle $\omega_{i(k)}$.

(i) For all $\epsilon, \epsilon>0$, an $R_{1}, R_{1}>R_{0}$, exists such that $R>R_{1}$ implies

$$
\left|\frac{1}{n-m} \omega_{i(k)}-C_{i(k)}^{R}\right|<\epsilon .
$$

(ii) In the situation of Figure $10 b$ we have that for each partition

$$
\omega_{i(k)}=\omega_{i(k)}^{\prime}+\omega_{i(k)}^{\prime \prime} \quad \text { with } \omega_{i(k)}^{\prime}, \omega_{i(k)}^{\prime \prime}>0 \text {, }
$$

exactly one $z^{*} \in \operatorname{arc}(R)$ exists such that the trajectory of $\overline{\mathcal{N}}(f)$ through $z^{*}$ (and tending to $\left.\omega_{i}\right)$ subdivides the angle $\omega_{i(\mathrm{k})}$ into two angles namely $\omega_{i(\mathrm{k})}^{\prime}$ and 
$\omega_{i(k)}^{\prime \prime}, \omega_{i(k)}^{\prime}$ being measured in an anticlockwise sense from the $\bar{G}(f)$-edge containing $\sigma_{i(k)}^{(1)}$ to this trajectory.

Proof.

(A similar property holds in the situation of Figure 10c.)

(i) It is not hard to see - use the Relation (2.1), the fact that $f$ is conformal at $\omega_{i}$ and the very definition of $\operatorname{arc}_{i(k)}^{(R)}-$ that

$$
\omega_{i(k)}=\operatorname{Im}\left[\frac{1}{2 \pi} \int_{\operatorname{arc}_{i(k)}} \frac{f^{\prime}(z)}{f(z)} \mathrm{d} z\right] .
$$

Note that for

$$
|z|>R_{0}: \frac{f^{\prime}(z)}{f(z)}=\frac{n-m}{z}+o\left(\frac{1}{z}\right) .
$$

This is easily seen, e.g., by considering the Laurent series of

$$
\frac{f^{\prime}(z)}{f(z)} \text { on }\left\{z|| z \mid>R_{0}\right\} \text {. }
$$

The assertion follows immediately (by estimating the integral).

(ii) Since $\omega_{i}$ is a stable proper node of $\overline{\mathcal{N}}(f)$, exactly one trajectory of $\overline{\mathcal{N}}(f)$ exists which exhibits the partition of $\omega_{i(k)}$. Since each trajectory in $A_{i(k)}$ intersects $\operatorname{arc}_{i(k)}^{(R)}$ exactly once $-C_{R}$ being global boundary - the point $z^{*}$ is uniquely determined.

Now, we turn over to the main result of this section, namely the converse of Lemma 4.2. We need some more definitions:

Given two connected plane graphs $\bar{G}_{1}, \bar{G}_{2}$ for which the angles are welldefined. Suppose that: $\bar{G}_{1} \sim \bar{G}_{2}$, the isomorphism being induced by the orientation preserving homeomorphism $\psi$. Let $\omega_{i(k)}$ be an angle for $\bar{G}_{1}$ between the $\bar{G}_{1}$-edges $x_{1}$ and $x_{2}$ containing $\omega_{i}$-measured from $x_{1}$ to $x_{2}$. Then, the angle between the $\bar{G}_{2}$-edges $\psi\left(x_{1}\right)$ and $\psi\left(x_{2}\right)$ - measured from $\psi\left(x_{1}\right)$ to $\psi\left(x_{2}\right)$ - is denoted by $\psi^{*}\left(\omega_{i(k)}\right)$.

DEFINITION 4.2. Let $\epsilon>0$ be arbitrary. The function $f \in \tilde{\mathscr{R}}_{+}$is said to 'realize the Newton graph $\bar{G}$ w.r.t. $\epsilon$ ' if an orientation preserving homeomorphism $\psi$ : $\mathbb{C} \rightarrow \mathbb{C}$ exists such that

(i) $\psi$ induces an isomorphism $\bar{G} \sim \bar{G}(f)$

(ii) $\left|\omega_{i(k)}-\psi^{*}\left(\omega_{i(k)}\right)\right|<\epsilon$, for all angles $\omega_{i(k)} \in A(\bar{G})$.

THEOREM 4.1 ( $\epsilon$-Representation Theorem). Let $\bar{G}$ be a Newton graph and let $\epsilon>0$ be given. Then, a function $f \in \tilde{\mathscr{R}}_{+}$exists which realizes $\bar{G}$ with respect to $\epsilon$.

COROLLARY 4.2 (Representation Theorem). Given an arbitrary Newton graph $\bar{G}$. Then, there exists an $f \in \tilde{R}_{+}$such that $\bar{G} \sim \bar{G}(f)$. 


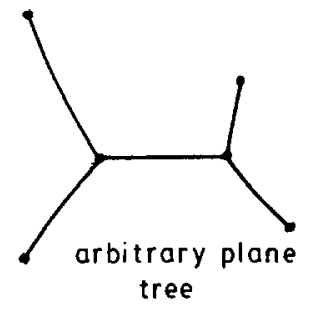

(a)

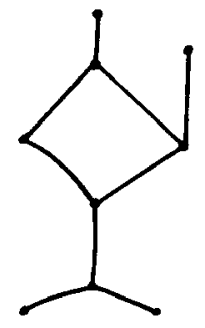

(b)

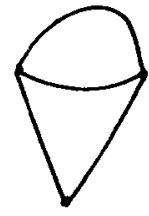

(c)

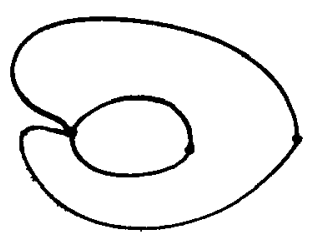

(d)

Fig. 11.

A function $f \in \tilde{R}_{+}$is said to realize a given plane graph $\bar{G}$ if $\bar{G} \sim \bar{G}(f)$. From Lemma 4.2 , Corollary 4.2 and Remark 4.1 it follows that plane graphs as depicted in Figure 11 may be realized by a function in $\tilde{\mathscr{R}}_{+}$, whereas this is impossible in the case of the plane graphs as depicted in Figure 12.

Proof (of Theorem 4.1). We give the proof by induction on $n(=$ order of $\bar{G}$ ) and $\mu(=$ cyclomatic number of $\bar{G})$.

Step 1. Suppose that $\mu=0$, so $\bar{G}$ is a tree. If $n=2$, then the function $f(z)=\left(z-\omega_{1}\right)\left(z-\omega_{2}\right)$, with $w_{1} \neq \omega_{2}$, realizes $\bar{G}$ w.r.t. (any) $\epsilon>0$.

Under the assumption that the assertion is true for trees of order $n, n \geqslant 2$, we consider a (Newton) tree $\bar{G}$ of order $(n+1)$.

Graph $\bar{G}^{0}$ is a plane tree which is obtained from $\bar{G}$ by removing all points of a $\bar{G}$-edge, say $x$, corresponding with a vertex of degree 1 , with the exception of the $x$-vertex which has degree $>1$ (use the fact that $n \geqslant 2$ ). Obviously, $\bar{G}^{0}$ is a Newton tree of order $n$.

The vertices (angles) of $\bar{G}$ are denoted by $\omega_{i}\left(\omega_{i(k)}\right), i=1, \ldots, n+1 ; k=$ $1, \ldots, \operatorname{deg} \omega_{i}$. The labelling is arranged in such a way that $x$ is the edge determined by $\omega_{n}$ and $\omega_{n+1}$, deg $\omega_{n+1}=1$ (thus deg $\omega_{n}>1$ ). The $\bar{G}$-angle at $\omega_{n}$ between $x$ and the edge preceding (proceeding) $x$ w.r.t. the anticlockwise order induced by the embedding of $\bar{G}$ is denoted by $\omega_{n\left(k_{0}\right)}\left(\omega_{n\left(k_{0}+1\right)}\right), k_{0}=\operatorname{deg} \omega_{n}-1$. It follows that $\tilde{\omega}_{n\left(k_{0}\right)}:=\omega_{n\left(k_{0}\right)}+\omega_{n\left(k_{0}+1\right)}$ is an angle for $\bar{G}^{0}\left(\right.$ at $\left.\omega_{n}\right)$. For the other

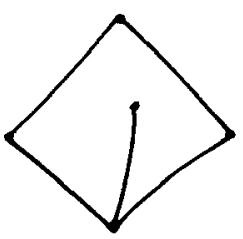

(a)

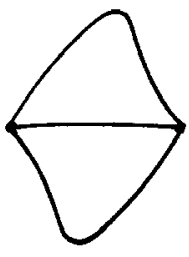

(b)

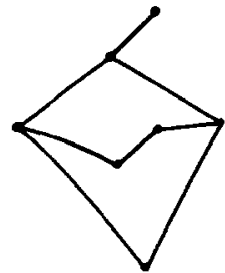

(c)

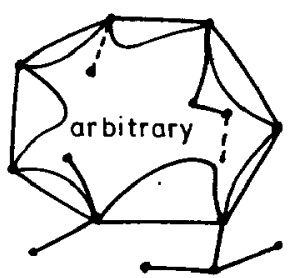

(d)

Fig. 12. 
$\bar{G}^{0}$-angles, denoted by $\tilde{\omega}_{i(k)}$, we find

$$
\begin{aligned}
& \tilde{\omega}_{i(k)}=\omega_{i(k)}, \quad i=1, \ldots, n ; \quad k=1, \ldots, d(i) ; i(k) \neq n\left(k_{0}\right) ; d(i)=\operatorname{deg} \omega_{i} \quad \text { if } \\
& i \neq n ; d(n)=k_{0} .
\end{aligned}
$$

Without loss of generality we may assume that

$$
0<\epsilon<\min A(\bar{G}),
$$

where min $A(\bar{G})$ stands for the minimum taken over all angles for $\bar{G}$.

By induction-assumption, $\bar{G}^{0}$ is realized w.r.t. $\epsilon / 3$ by a polynomial $p$ in $\tilde{R}_{+}$of degree $n$, by means of an orientation preserving homeomorphism $\psi: \mathbb{C} \rightarrow \mathbb{C}$. We shall construct from $p$ a polynomial, again in $\tilde{R}_{+}$, of degree $(n+1)$ which realizes $\overline{\mathscr{G}}$ with respect to $\epsilon$.

Obviously, we have $N(p)=\left\{\psi\left(\omega_{i}\right) \mid i=1, \ldots, n\right\}$, and

$$
\left|\psi^{*}\left(\tilde{\omega}_{i(k)}\right)-\tilde{\omega}_{i(k)}\right|<\frac{\epsilon}{3}, \quad i=1, \ldots, n ; k=1, \ldots, d(i) .
$$

We choose $R_{0}, R_{0}>0$, such that for all $R$, with $R>R_{0}$

$N(p) \cup C(p)$ is contained in the disk $D_{R}:=\{z|| z \mid \leqslant R\}$,

The circle $C_{R}:=\{z|| z \mid=R\}$ is global boundary for $\overline{\mathcal{N}}(p)$.

Let $R, R>R_{0}$ be arbitrary but fixed.

Since $p$ is a polynomial (thus $p$ has no finite poles) all canonical regions of $p$ are of the types as depicted in Figure 10b,c. Consequently, with respect to $p$, the $\operatorname{arcs} \operatorname{arc}_{i(k)}^{(R)}$ are well-defined, $i=1, \ldots, n ; k=1, \ldots, d(i)$. See also Figure 13 .

Let $z^{*} \in \operatorname{arc}_{n\left(k_{0}\right)}^{(R)}$ be the (uniquely determined) point such that the $\overline{\mathcal{N}}(p)$ trajectory through $z^{*}$ subdivides the $\bar{G}(p)$-angle $\psi^{*}\left(\tilde{\omega}_{n\left(k_{0}\right)}\right)$ into the angles $\alpha_{1}, \alpha_{2}$ (the order being in accordance with the anticlockwise ordering on the angles at

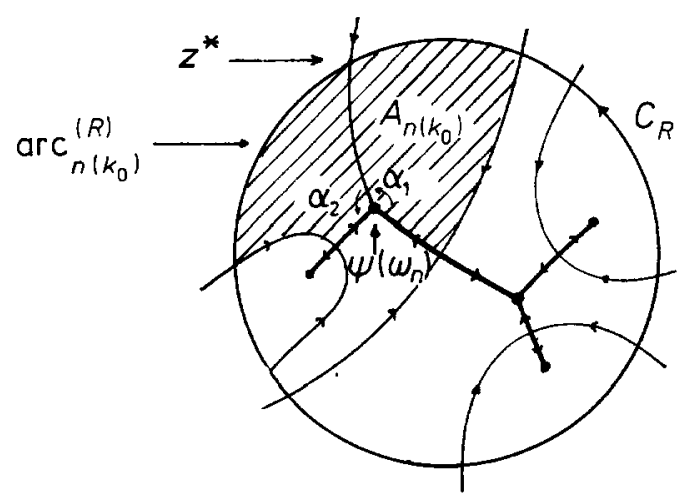

Fig. 13. 
$\left.\psi\left(\omega_{n}\right)\right)$ such that

$$
\alpha_{1}=\omega_{n\left(k_{1}\right)}, \quad \alpha_{2}=\psi^{*}\left(\tilde{\omega}_{n\left(k_{0}\right)}\right)-\omega_{n\left(k_{0}\right)} .
$$

Such $z^{*}$ does always exist as a consequence of Lemma 4.3(ii); note that in view of (1) and (2), we have $\alpha_{2}>0$.

From (2) we obtain

$$
\left|\alpha_{2}-\omega_{n\left(k_{1}+1\right)}\right|<\frac{\epsilon}{3} .
$$

The angles spanned by the arcs into which $z^{*}$ subdivides $\operatorname{arc}_{n\left(k_{1}\right)}^{(R)}$ are denoted by $2 \pi c_{1}, 2 \pi c_{2}, c_{1}, c_{2}>0$ (the order being in accordance with the positive orientation of $C_{R}$ ). We define: $\Theta=\min \left\{2 \pi c_{1}, 2 \pi c_{2}\right\}$.

For $\eta>0$ and $\nu \in \mathbf{R}$ we define the polynomials $q_{\eta, \nu}$ as follows

$$
q_{\eta, \nu}(z)=\left(-\eta \mathrm{e}^{-i \nu} z+1\right) p(z)
$$

As a consequence of the forthcoming Lemma 4.4(i), we have

Taking $\eta$, with $\eta>0$, sufficiently small, the vector field $\left.\overline{\mathcal{N}} q_{\eta, v}\right) \mid D_{R}$ will be - uniformly in $\nu$-arbitrary $C^{1}$-close to $\overline{\mathcal{N}}(p) \mid D_{R}$.

Since $p \in \tilde{\mathscr{R}}_{+}$and $C_{R}$ is a global boundary for $\overline{\mathcal{N}}(p)$, it follows from Theorem 3.1 and the characterization theorem of de Baggis and Peixoto on structural stability in the plane (cf. Section 3):

The system $\overline{\mathcal{N}}(p) \mid D_{R}$ is $\epsilon$-structurally stable.

From (6) and (7) it follows that an $\eta_{1}, 0<\eta_{1}<1 / R$, exists such that for all $\eta$, $0<\boldsymbol{\eta}<\boldsymbol{\eta}_{1}$, and all $\nu \in \mathbf{R}$ there are orientation preserving homeomorphisms $\Psi_{\eta, \nu}: D_{R} \rightarrow D_{R}$ fulfilling the following properties:

$C_{R}$ is global boundary for $\overline{\mathcal{N}}\left(q_{\eta, \nu}\right)$, all $\nu \in \mathbf{R}$

The (maximal) trajectories of $\overline{\mathcal{N}}(p) \mid D_{R}$ are mapped by $\Psi_{\eta, \nu}$ onto (maximal) trajectories of $\overline{\mathcal{N}}\left(q_{\eta, \nu}\right) \mid D_{R}$; in particular zeros and critical points for $p$ are mapped to zeros and critical points for $q_{\eta, \nu}$, respectively.

For all $z \in D_{R}$ and all $\nu \in \mathbf{R}$, we have $\left|\Psi_{\eta, \nu}(z)-z\right|<\delta_{1}$, where

$$
\delta_{1}=\min \left\{\frac{R \cdot \Theta}{3},\left|\psi\left(\omega_{i}\right)-\psi\left(\omega_{i^{\prime}}\right)\right| ; i, i^{\prime}=1, \ldots, n ; i \neq i^{\prime}\right\}
$$

Note that the maps $\Psi_{\eta, \nu}$ are orientation preserving in two senses:

- Any positively oriented closed Jordan curve in $D_{R}$ is mapped by $\Psi_{\eta, \nu}$ onto such a curve.

- The homeomorphisms $\Psi_{\eta, \nu}$ map trajectories onto trajectories respecting the orientations of these trajectories.

The properties (8), (9) and (10) yield the following conclusions which hold for all 
$\eta$ with $0<\eta<\eta_{1}$ and for all $\nu \in \mathbf{R}$ :

(a) The $n$ different zeros for $p$ are fixed points for $\Psi_{\eta, \nu}$. In fact, the zeros for $p$ are also zeros for $q_{\eta, \nu}$.

(b) The polynomials $q_{\eta, \nu}$ have $(n-1)$ simple critical points inside $C_{R}$.

(c) The polynomials $q_{\eta, \nu}$ have exactly one zero (namely $\left.1 / \eta\right) \mathrm{e}^{i \nu}$ ) and exactly one simple critical point, denoted $\sigma_{\eta, \nu}$, on $\mathbb{C} \mid D_{R}$.

(d) An unstable $\overline{\mathcal{N}}(p)$-manifold (at a critical point for $p$ ) which 'connects' $\psi\left(\omega_{i}\right)$ and $\psi\left(\omega_{i}\right), i \neq i^{\prime}$, is mapped under $\Psi_{\eta, \nu}$ onto an unstable $\overline{\mathcal{N}}\left(q_{\eta, \nu}\right)$ manifold which also 'connects' $\psi\left(\omega_{i}\right)$ and $\psi\left(\omega_{i}\right.$ ').

(e) The $\overline{\mathcal{N}}\left(q_{\eta, \nu}\right)$-trajectory through $z^{*}$ is regular and tends to $\psi\left(\omega_{n}\right)$.

If we denote by $\gamma_{+}\left(\sigma_{\eta, v}\right), \gamma_{-}\left(\sigma_{\eta, v}\right)$, respectively, the stable, unstable manifold at $\sigma_{\eta, \nu}$ of $\overline{\mathcal{N}}\left(q_{\eta, \nu}\right)$, then (a)-(d) yield (cf. Figure 14):

(f) $\gamma_{+}\left(\sigma_{\eta, \nu}\right) \cap C_{R}=\emptyset$.

(g) One of the separatrices in $\gamma_{-}\left(\sigma_{\eta, \nu}\right)$ tends to $(1 / \eta) \mathrm{e}^{i \nu}$ and does not cross $C_{R}$. The other separatrix in $\gamma_{-}\left(\sigma_{\eta, \nu}\right)$ crosses $C_{R}$ only once and tends to a zero or to a critical point for $q_{\eta, \nu}$ inside $C_{R}$. (Use also Lemma 2.1.)

(h) The polynomials $q_{\eta, \nu}$ are nondegenerate iff the only separatrix in $\gamma_{-}\left(\sigma_{\eta, \nu}\right)$ which crosses $C_{R}$ tends to a zero for $p$. (Use also (f), (g).)

From now on we restrict ourselves to $\eta$ with $0<\eta<\eta_{1}$.

We define the plane graph $\bar{G}_{(\eta, v)}^{\circ}$ as follows:

- The vertices of $\bar{G}_{(\eta, \nu)}^{\circ}$ are all zeros for $q_{\eta, \nu}$ inside $C_{R}$.

- The edges of $\bar{G}_{(\eta, \nu)}^{\circ}$ are all (topological) closures of the unstable $\overline{\mathcal{N}}\left(q_{\eta, \nu}\right)$ manifolds at the critical points for $q_{\eta, \nu}$ inside $C_{R}$.

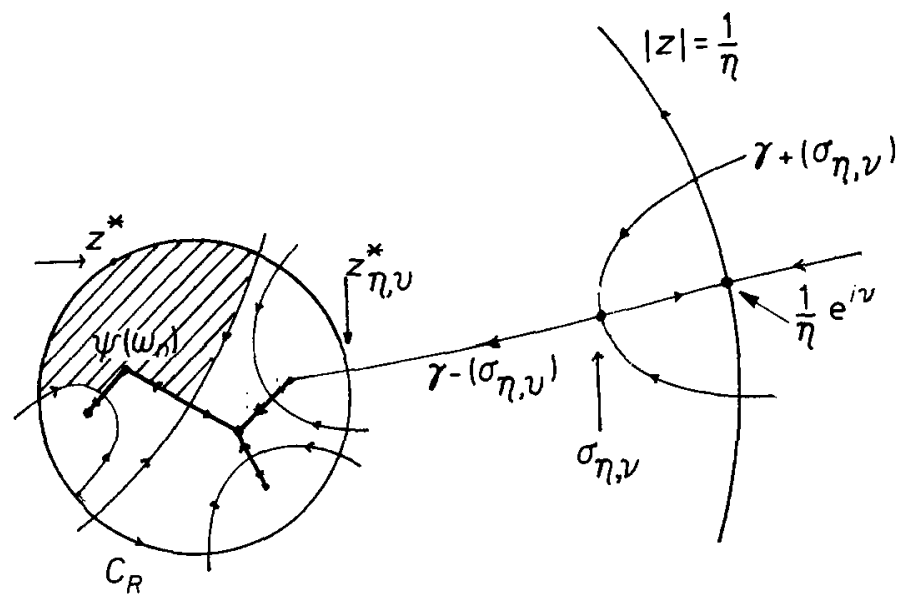

Fig. 14. 
In view of (a) and (d) it follows that $\bar{G}(p) \sim \bar{G}_{(\eta, v)}^{\circ}$, the graph isomorphism being induced by $\Psi_{\eta, \nu}$. Consequently, $\bar{G}_{(\eta, \nu)}^{\circ}$ is a plane tree.

Obviously, the angles for $\bar{G}_{(\eta, \nu)}^{\circ}$ are well-defined.

The angle of $\bar{G}_{(\eta, \nu)}^{o}$ corresponding (under $\left.\Psi_{\eta, \nu}\right)$ with the $\bar{G}(p)$-angle $\psi^{*}\left(\tilde{\omega}_{i(k)}\right)$ is denoted by

$$
\Psi_{\eta, \nu}^{*} \circ \psi^{*}\left(\tilde{\omega}_{i(k)}\right), \quad i=1, \ldots, n ; k=1, \ldots, \mathrm{d}(i) .
$$

In the case that $\mathrm{d}(i)=1$ we have:

$$
\psi^{*}\left(\tilde{\omega}_{i(1)}\right)=\Psi_{\eta, \nu}^{*} \circ \psi^{*}\left(\tilde{\omega}_{i(1)}\right)=1 .
$$

If $\mathrm{d}(i)>1$ then Lemma 4.1 and (d) yield

$$
\psi^{*}\left(\tilde{\omega}_{i(k)}\right)-\Psi_{\eta, \nu}^{*} \psi^{*}\left(\tilde{\omega}_{i(k)}\right)=\frac{1}{2 \pi}\left[\arg \frac{p\left(\sigma_{i(k)}^{(2)}\right)}{p\left(\sigma_{i(k)}^{(1)}\right)}-\arg \frac{q_{\eta, \nu}\left(\Psi_{\eta, \nu}\left(\sigma_{i(k)}^{(2)}\right)\right.}{q_{\eta, \nu}\left(\Psi_{\eta, \nu}\left(\sigma_{i(k)}^{(1)}\right)\right.}\right] .
$$

In view of the $\epsilon$-structural stability of $\overline{\mathcal{N}}(p) \mid D_{R}$ and (6) it follows from (11) that, uniformly in $\nu$ :

$$
\lim _{\eta \downarrow 0}\left(\psi^{*}\left(\tilde{\omega}_{i(k)}\right)-\Psi_{\eta, \nu}^{*} \circ \psi^{*}\left(\tilde{\omega}_{i(k)}\right)\right)=0, \quad i=1, \ldots, n ; k=1, \ldots, \mathrm{d}(i) .
$$

We conclude that an $\eta_{2}$ with $0<\eta_{2}<\eta_{1}$ exists such that for all $\eta$, with $0<\eta<\eta_{2}$, and all $\nu \in \mathbf{R}$ we have:

$$
\bar{G}_{(\eta, \nu)}^{\circ} \text { is realized by } p \text { with respect to } \frac{\epsilon}{3} \text {. }
$$

Suppose $0<\eta<\eta_{2}$.

Consider the $\overline{\mathcal{N}}\left(q_{\eta, \nu}\right)$-trajectory through $z^{*}$. This trajectory subdivides the $\bar{G}_{(\eta, \omega)}^{\circ}$-angle $\Psi_{\eta, \nu}^{*}{ }^{\circ} \psi^{*}\left(\tilde{\omega}_{n\left(k_{0}\right)}\right)$ into the nonvanishing angles $\beta_{(\eta, \nu)}^{1}, \beta_{(\eta, \nu)}^{2}$, the order being in accordance with the anticlockwise ordering on the set of angles at $\psi\left(\omega_{n}\right)$. This is an easy consequence of (e).

Using essentially the same techniques as needed for (11) and (12) we derive

There exists an $\eta_{3}$, with $0<\eta_{3}<\eta_{2}$ such that:

$$
\left|\alpha_{i}-\beta_{(\eta, v)}^{i}\right|<\frac{\epsilon}{3}, \quad i=1,2 ; \text { all } \nu \in \mathbf{R}
$$

From (12) and (13), the induction assumption on $\bar{G}^{\circ}$ and (e), (h), it follows that $\bar{G}$ is realized w.r.t. $\epsilon$ by the nondegenerate polynomial $q_{\eta, \nu_{0}}, 0<\eta<\eta_{3}$, if we are able to prove that an $\nu_{0} \in \mathbf{R}$ exists such that $\gamma_{-}\left(\sigma_{\eta, \nu_{0}}\right)$ contains $z^{*}$. The existence of such $\nu_{0}$ is shown as follows

Let $\eta$ with $0<\eta<\eta_{3}$, be chosen.

In view of $(\mathrm{g})$ it follows that $\gamma_{-}\left(\sigma_{\eta, \nu}\right)$ has exactly one point in common with $C_{R}$, say $z_{\eta, \nu}^{*}$. Consequently, the following map is well-defined (see Figure 14). 


$$
\chi_{1}:\left\{z|| z \mid=\frac{1}{\eta}\right\} \rightarrow C_{R} \quad \text { where } \chi_{1}\left(\frac{1}{\eta} \mathrm{e}^{\mathrm{i} \nu}\right)=z_{\eta, \nu}^{*} .
$$

In Lemma 4.5.(i) we shall prove that $\chi_{1}$ is surjective. This concludes the proof of Step 1.

Step 2. Suppose that the theorem is true for Newton graphs with cyclomatic number $m$ with $m \geqslant 0$ arbitrary. We prove that, under this assumption, the theorem is also true for a Newton graph $\bar{G}$ with $\mu(\bar{G})=m+1$.

Since this proof, for a large part, runs along the same lines as the proof exposed under Step 1, we just focus our attention to those aspects which are different.

As usual we denote the regions of $\bar{G}$ by $r_{0}, r_{1}, \ldots, r_{m+1}$ where $r_{0}$ is the unbounded region.

Let $x$ be a $\bar{G}$-edge with $x \in \partial r_{0} \cap \partial r_{k_{k}}$, some $j_{0} \in\{1, \ldots, m+1\}$. Such $x$ always exists, since $\mu(\bar{G})=m+1>0$.

If we delete from $\bar{G}$ all points of $x$ with the exception of the $\bar{G}$-vertices in $x$, then we obtain a Newton graph $\bar{G}^{\circ}$.

Obviously we have $\mu\left(\bar{G}^{\circ}\right)=m$ and order $\bar{G}^{\circ}=n$.

Without loss of generality we assume that $j_{0}=m+1$, thus $r_{1}, \ldots, r_{m}$ are the bounded regions of $\bar{G}^{\circ}$.

The orientation of the Eulerian trail $\tau_{m+1}$ of $\partial r_{m+1}$ induces an orientation on $x$. (Recall the uniqueness of the construction of $\tau_{m+1}$ in view of the fact that $\bar{G}$ is a Newton graph.) The labeling of the $\bar{G}$-vertices $\omega_{i}, i=1, \ldots, n$ is arranged in such a way that $\omega_{1}\left(\omega_{2}\right)$ is the begin (end) vertex of $x$. By $\omega_{1(2)}, \omega_{2(1)}$ we denote the angle of $\tau_{m+1}$ between $x$ and the edge preceding (proceeding) $x$ in $\tau_{m+1}$. See Figure 15.

The angles of $\tau_{m+1}$ are denoted by $\phi_{1}\left(=\omega_{1(2)}\right), \phi_{2}\left(=\omega_{2(1)}\right), \phi_{3}, \ldots, \phi_{s}$, the order being in accordance with the orientation of $\tau_{m+1}$. Thus, $s=$ the number of edges in $\tau_{m+1}$; since there are no loops in $\bar{G}$, we have $s \geqslant 2$.

Without loss of generality we may assume

$$
0<\epsilon<\min A(\bar{G}) \text {. }
$$

By induction assumption, an $f$ in $\tilde{\mathscr{R}}_{+}$(again represented by $p_{n} / q_{m}$ ) exists which realizes $\bar{G}^{\circ}$ with respect to $\epsilon / 6 s$ by means of the (orientation preserving)

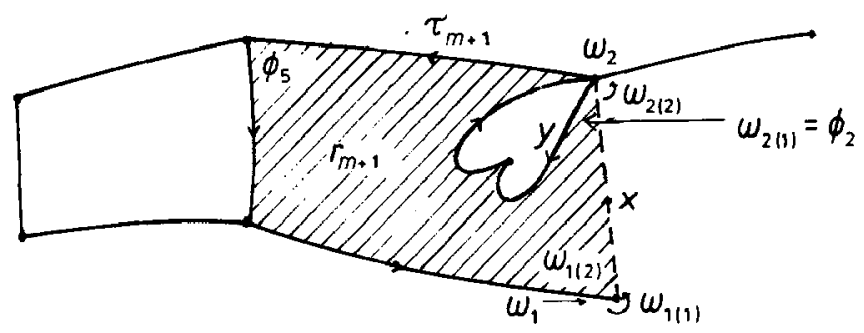

Fig. 15. 
homeomorphism $\psi: \mathbb{C} \rightarrow \mathbb{C}$

Since $f \in \tilde{R}_{+}$an $\boldsymbol{R}_{\mathbf{0}}, \boldsymbol{R}_{\mathbf{0}}>0$, exists such that for all $\boldsymbol{R}>\boldsymbol{R}_{\mathbf{0}}$ :

$$
N(f) \cup P(f) \cup C(f) \subset D_{R},
$$

$C(R)$ is global boundary for $\overline{\mathcal{N}}(f)$.

Let $R, R>R_{0}$, be arbitrary. In the case that $s>2$, the $\bar{G}$-angles $\phi_{l}, l=3, \ldots, s$, are also $\overline{\mathscr{G}}^{\circ}$-angles. By $\Lambda$ we denote the union of all canonical regions $-A_{\phi_{1}}-$ of $\overline{\mathcal{N}}(f)$ determined by the angles $\psi^{*}\left(\phi_{l}\right), l=3, \ldots, s$. If $s=2$, we define $\Lambda=\emptyset$. All canonical regions $A_{\phi_{1}}, l \neq 1,2$, are of the form as depicted in Figure $10 \mathrm{~b}$.

One easily verifies that, in the case $s \geqslant 4$, each intersection $\bar{A}_{\phi_{l}} \cap \bar{A}_{\phi_{l+1}}$, is just the closure of one of the trajectories in the stable $\overline{\mathcal{N}}(f)$-manifold at the critical point for $f$ on the $\bar{G}(f)$-edge between the zeros for $f$ corresponding with $\phi_{l}$ and $\phi_{l+1} ; l=3, \ldots, s-1$. See Figure 16. From this it follows (use (3')) that $\bar{\Lambda} \cap C_{R}$ is a closed connected arc (eventually empty), denoted by $\operatorname{arc}^{(R)}(\Lambda)$. The $\bar{G}(f)$ angles $\psi^{*}\left(\omega_{1(1)}+\omega_{1(2)}\right), \psi^{*}\left(\omega_{2(1)}+\omega_{2(2)}\right)$ determine the canonical regions $A_{1}, A_{2}$. These regions are of the form as depicted in Figures $10 \mathrm{~b}$ or $\mathrm{c}$. The $\operatorname{arc} A_{1} \cap C_{R}$ is oriented in accordance with the positive orientation of $C_{R}$; the begin(end)-point of this arc is denoted by $\lambda_{1}^{(R)}\left(\lambda_{2}^{(R)}\right)$; see Figure 16 .

We define: $\Lambda^{\prime}=A_{1} \cup \Lambda \cup A_{2}$. One easily checks that $\bar{\Lambda}^{\prime} \cap C_{R}$ is a closed, connected, nonempty arc. This arc is denoted by $\operatorname{arc}^{(R)}\left(\Lambda^{\prime}\right)$.

As in Step 1, we can choose in the $\operatorname{arc}\left(A_{2} \cap C_{R}\right)$ an $z_{(R)}^{*}$ such that the $\overline{\mathcal{N}}(f)$-trajectory through $z_{(R)}^{*}$ subdivides the $\bar{G}(f)$-angle $\psi^{*}\left(\omega_{2(1)}+\omega_{2(2)}\right)$ at $\psi\left(\omega_{2}\right)$ into any two nonvanishing angles $\alpha_{1}, \alpha_{2}$. We choose $\alpha_{1}=\omega_{2(1)}\left(=\phi_{2}\right)$, where this angle is measured in an anticlockwise sense from $\psi(y), y=(\bar{G}$-edge proceeding $x$

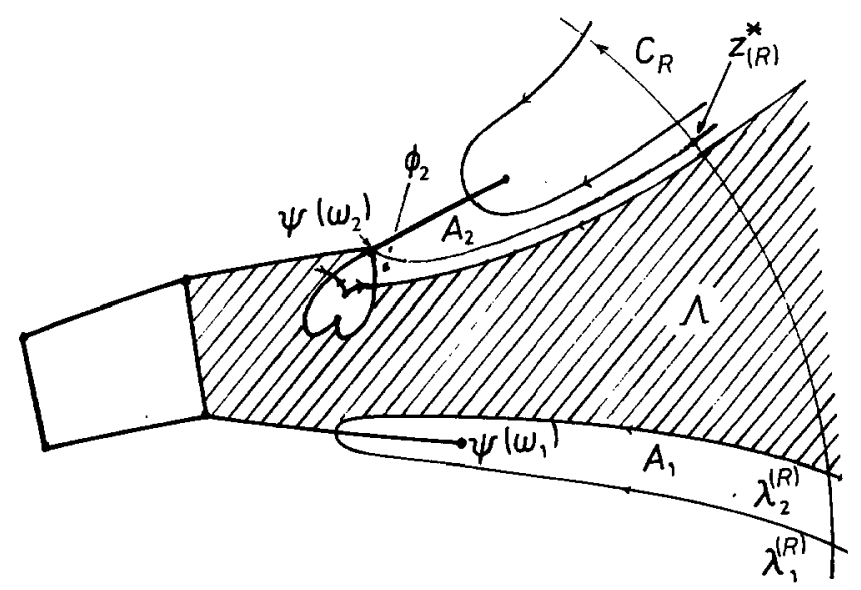

Fig. 16. 
in $\left.\tau_{m+1}\right)$, to this trajectory. The positively oriented arc with beginpoint $\lambda_{i}^{(R)}$ and endpoint $z_{(R)}^{*}$ is spanned by a positive angle, say $2 \pi c^{(R)}\left(\lambda_{i}, z^{*}\right) ; i=1,2$.

We are going to fix $R$ in a suitable way.

Since $\bar{G}$ is a Newton graph and $\bar{G}^{\circ}$ is realized by $f$ w.r.t. $\epsilon / 6 s$ we have

$$
\begin{aligned}
& \left|\left(\sum_{l=3}^{s} \psi^{*}\left(\phi_{l}\right)+\phi_{2}\right)-\left(1-\phi_{1}\right)\right|<\frac{\epsilon}{6}, \\
& \left|\left(\sum_{l=3}^{s} \psi^{*}\left(\phi_{l}\right)+\phi_{2}+\psi^{*}\left(\omega_{1(1)}+\omega_{1(2)}\right)\right)-\left(1+\omega_{1(1)}\right)\right|<\frac{\epsilon}{6}
\end{aligned}
$$

where $\sum_{l=3}^{s} \psi^{*}\left(\phi_{l}\right):=0$ if $s=2$.

There exists an $R_{1}$ with $R_{1}>R_{0}$ such that for all $R$ with $R>R_{1}$

$$
\begin{aligned}
& c^{(R)}\left(\lambda_{1}, z^{*}\right)>\frac{1}{n-m} \cdot\left(1+\omega_{1(1)}-\frac{\epsilon}{3}\right), \\
& c^{(R)}\left(\lambda_{2}, z^{*}\right)<\frac{1}{n-m} \cdot\left(1-\omega_{1(2)}+\frac{\epsilon}{3}\right) .
\end{aligned}
$$

This follows from (14) and from Lemma 4.3(i) as well as from techniques as used in the proof of latter lemma.

Put $\Phi(z)=z^{-n+m} f(z)$. Without loss of generality, we may assume that $\lim _{z \rightarrow \infty} \Phi(z)=-1$. Consequently, there exists an $R_{2}$ with $R_{2}>R_{0}$, such that for all $R>R_{2}$ we have arg $\Phi(z)$ depends continuously on $z$ if $z$ traverses the circle $C_{R}$ and, moreover (for all $z \in C_{R}$ ):

$$
|\arg \Phi(z)-\pi|<\frac{\epsilon}{10}
$$

We fix $R$ such that $R>\max \left(R_{1}, R_{2}\right)$.

We consider, for all $\eta>0, \nu \in \mathbf{R}$, the functions

$$
g_{\eta, \nu}(z)=\left(-\eta \mathrm{e}^{-i \nu} z+1\right)^{-1} f(z) \text {. }
$$

Since $\bar{G}$ is a Newton graph and thus $m+1<n$, we have $g_{\eta, \nu} \in \mathscr{R}_{+}$.

Properties (6) and (7) of Step 1 also hold if $f\left(g_{\eta, \nu}\right)$ plays the role of $p\left(q_{\eta, \nu}\right)$ in Step 1 . We refer to these properties as $\left(6^{\prime}\right)$ and $\left(7^{\prime}\right)$. The validity of $\left(6^{\prime}\right)$ follows from the forthcoming Corollary 4.3 , whereas $\left(7^{\prime}\right)$ follows from the facts that $f \in \tilde{\mathscr{R}}$ and $C_{R}$ is global boundary of $\overline{\mathcal{N}}(f)$.

Although it will not be explicitly stated, the following properties and statements hold (as in the case of their analogues in Step 1) for all $\nu \in \mathbf{R}$ and for all positive, sufficiently small $\eta$.

In analogy to (c) in Step 1 we have:

(c') On $\mathbb{C} \mid D_{R}$, the functions $g_{\eta, \nu}$ have exactly one simple finite pole (namely $\left.(1 / \eta) \mathrm{e}^{i \nu}\right)$, one simple critical point (denoted $\sigma_{\eta, \nu}$ ) and no zeros.

Moreover: all critical points for $g_{\eta, \nu}$ are simple; $N(f)=N\left(g_{\eta, \nu}\right)$. 
As in Step 1, we define the plane graph $\bar{G}_{(\eta, \nu)}^{0}$ as follows:

- The vertices of $\bar{G}_{(\eta, \nu)}^{\circ}$ are the zeros for $g_{\eta, \nu}$ inside $C_{R}$.

- The edges of $\bar{G}_{(\eta, \nu)}^{\circ}$ are the (topological) closures of the unstable $\overline{\mathcal{N}}\left(g_{\eta, \nu}\right)$ manifolds at the critical points for $g_{\eta, \nu}$ inside $C_{R}$.

As in Step 1 we have:

The function $f$ realizes $\bar{G}_{(\eta, \nu)}^{\circ}$ with respect to $\epsilon / 6 s$.

The graph isomorphism underlying $\bar{G}(f) \sim \bar{G}_{(\eta, \nu)}^{\circ}$ is induced by orientation preserving homeomorphic 'perturbations' $\Psi_{\eta, \nu}: D_{R} \rightarrow D_{R}$.

(The proof of $\left(12^{\prime}\right)$, which is essentially based on $\left(6^{\prime}\right)$ and $\left(7^{\prime}\right)$, runs along the same lines as the proof of (12) and will be omitted.)

The stable (unstable) $\overline{\mathcal{N}}\left(g_{\eta, \nu}\right)$-manifold at $\sigma_{\eta, \nu}$ is denoted by $\gamma_{+}\left(\sigma_{\eta, \nu}\right)$ $\left(\gamma_{-}\left(\sigma_{\eta, \nu}\right)\right)$. Both separatrices in $\gamma_{-}\left(\sigma_{\eta, \nu}\right)$ cross the circle $C_{R}$ only once. This follows from the fact that $C_{R}$ is also a global boundary for $\overline{\mathcal{N}}\left(g_{\eta, \nu}\right)$, outside $C_{R}$ there are no critical points (different from $\sigma_{\eta, \nu}$ ) and no zeros for $g_{\eta, \nu}, z=\infty$ is a pole for $g_{\eta, \nu}$ and Lemma 2.1. (See also Figure 17).

The intersection points of $\gamma_{-}\left(\sigma_{\eta, \nu}\right)$ and $C_{R}$ determine two open arcs of $C_{R}$. For exactly one of these arcs the following property holds: the $\overline{\mathcal{N}}\left(g_{\eta, \nu}\right)$-trajectory through any of its points emanates from $(1 / \eta) e^{i \nu}$.

We denote this arc by $\operatorname{arc}_{(\eta, \nu)}^{(R)}$ and orient it in accordance with the positive orientation of $C_{R}$. By $z_{\eta, \nu}^{*}\left(z_{\eta, \nu}^{* *}\right)$ we denote the end (begin) point of $\operatorname{arc}(\eta, \nu)$. The positive angle spanned by $\operatorname{arc}_{\eta, \nu)}^{(R)}$ is denoted by $2 \pi c c_{\eta, \nu)}^{(R)}$.

Since for each suitable pair $(\eta, \nu)$ the point $z_{\eta, \nu}^{*}$ is uniquely determined, the

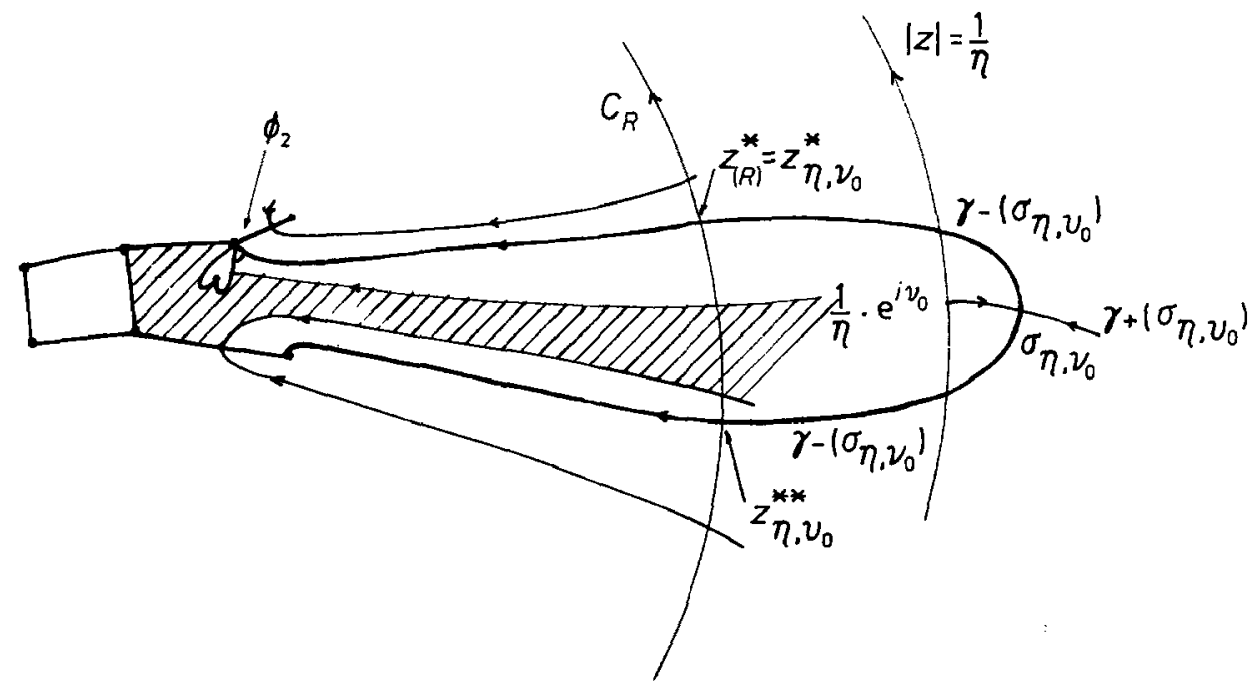

Fig. 17. 
following map ( $\eta$ fixed) is well-defined:

$$
\chi_{2}:\left\{z|| z \mid=\frac{1}{\eta}\right\} \rightarrow C_{R} \quad \text { where } \chi_{2}\left(\frac{1}{\eta} \mathrm{e}^{i \nu}\right)=z_{\eta, \nu}^{*} .
$$

It turns out (see Lemma 4.5) that $\chi_{2}$ is surjective. Therefore, we may choose $\nu_{0} \in \mathbf{R}$ such that $z_{\eta, \nu_{0}}^{*}=z_{(R)}^{*}$. (Note that $\nu_{0}$ depends on the choice of $\eta$.) As in Step 1 the separatrix in $\gamma_{-}\left(\sigma_{\eta, \nu_{0}}\right)$ through $z_{(R)}^{*}$ tends to $\psi\left(\omega_{2}\right)$ and moreover for the angles $\boldsymbol{\beta}_{\left(\eta, \nu_{0}\right)}^{(1)}, \boldsymbol{\beta}_{\left(\eta, \nu_{1}\right)}^{(2)}$ into which this separatrix subdivides the $\overline{\boldsymbol{G}}_{\left(\eta, \nu_{0}\right)}^{\circ}$ angle at $\psi\left(\omega_{2}\right)$, we have:

$$
\left|\alpha_{i}-\beta_{\left(\eta, \nu_{i}\right)}^{(i)}\right|<\frac{\epsilon}{6 s}, \quad i=1,2 .
$$

Note that:

- If the separatrices in $\gamma_{-}\left(\sigma_{\eta, \nu}\right)$ tend to zeros for $g_{\eta, \nu}$ (inside $C_{R}$ ), then, in view of Relation (2.1), these zeros must be different.

- Since $C_{R}$ is a global boundary for $\overline{\mathcal{N}}\left(g_{\eta, \nu}\right)$ the separatrices in $\gamma_{+}\left(\sigma_{\eta, \nu}\right)$ cannot cross $C_{R}$. One easily shows that one of these separatrices emanates from $(1 / \eta) \mathrm{e}^{i v}$ and the other from $z=\infty$ (see Figure 17).

Similar to (h) in Step 1 we can prove:

$\left(h^{\prime}\right)$ The functions $g_{\eta, v}$ are nondegenerate iff both separatrices in $\gamma_{-}\left(\sigma_{\eta, v}\right)$ tend to zeros for $g_{\eta, \nu}$.

We are going to fix $\eta$ in a suitable way. Let $\eta_{1}, \eta_{1}>0$, be such that for all $\eta \in$ ] $0, \eta_{1}[$, the properties and statements above which depend on the choice of $\eta$ hold.

In view of $\left(6^{\prime}\right)$ and $\left(7^{\prime}\right)$ we may choose an $\eta_{2}, \eta_{2}<\eta_{1}$, such that, for all $z \in D_{R}$, $\nu \in \mathbf{R}$ and for all $\eta \in] 0, \eta_{2}[$, we have

$$
\left|\Psi_{\eta, \nu}(z)-z\right|<\frac{\pi R}{n-m} \cdot \frac{\epsilon}{6} \text {. }
$$

The positively oriented arc of $C_{R}$ with $\Psi_{\eta, \nu}\left(\lambda_{i}^{(R)}\right)$ as the beginpoint and $z_{(R)}^{*}$ as the endpoint is spanned by the positive angle $2 \pi \mathbf{c}_{\eta, \nu}^{(R)}\left(\lambda_{i}, z^{*}\right), i=1,2$. From (15) and (17) it follows (for all $\eta \in] 0, \eta_{2}[$ and all $\nu \in \mathbf{R}$ )

$$
\begin{aligned}
& c_{\eta, \nu}^{(R)}\left(\lambda_{1}, z^{*}\right)>\frac{1}{n-m}\left(1+\omega_{1(1)}-\frac{\epsilon}{2}\right) \\
& c_{\eta, \nu}^{(R)}\left(\lambda_{2}, z^{*}\right)<\frac{1}{n-m}\left(1-\omega_{1(2)}+\frac{\epsilon}{2}\right)
\end{aligned}
$$

If $z$ traverses $\operatorname{arc}(\eta, \nu)$ from $z_{\eta, \nu}^{* *}$ to $z_{\eta, \nu}^{*}$ then the argument of $g_{\eta, \nu}(z)$ increases continuously by $2 \pi$.

This follows from: (1) $(1 / \eta) \mathrm{e}^{i \nu}$ is a simple pole for $g_{\eta, \nu}$; (2) Relation (2.1); (3) Each $\overline{\mathcal{N}}\left(g_{\eta, \nu}\right)$-trajectory through $\operatorname{arc}_{(\eta, \nu)}^{(R)}$ emanates from $(1 / \eta) \mathrm{e}^{i \nu}$. 
Suppose that if $z$ traverses $\operatorname{arc}_{(\eta, v)}^{(R)}$ from $z_{\eta, \nu}^{* *}$ to $z_{\eta, \nu}^{*}$, the argument of $f(z)$ increases continuously by $\Delta_{\eta, \nu}$.

Since $\lim _{\eta \downarrow 0} g_{\eta, \nu}(z)=f(z)$ (uniformly in $\nu$ ) we have: $\lim _{\eta \downarrow 0} \Delta_{\eta, \nu}=2 \pi$ (uniformly in $\nu$ ). From this it follows (in view of (16)) that for $\eta(>0)$ sufficiently small and all $\nu$ :

$$
\left|c_{(\eta, v)}^{(R)}-\frac{1}{n-m}\right|<\frac{1}{2} \frac{\epsilon}{n-m} .
$$

We conclude (use also (18)) that, putting $\nu=\nu_{0}$, we may choose a number $\left.\eta_{3} \in\right] 0, \eta_{2}[$ such that

$$
z_{\eta_{3}, \nu_{0}}^{* *} \in \Psi_{\eta_{3}, \nu_{0}}\left(A_{1} \cap C_{\mathrm{R}}\right) \text {. }
$$

In view of the choice of $\eta_{3}$ and $\nu_{0},(19)$ and $h^{\prime}$ we have: $g_{\eta_{3}, \nu_{0}} \in \tilde{R}_{+}$. Consequently, the plane-graph $\overline{\mathscr{G}}\left(g_{\eta_{3}, \nu_{0}}\right)$ is well-defined. The bounded region of $\bar{G}\left(g_{\eta_{3}, \nu_{0}}\right)$ which is determined by $\left(1 / \eta_{3}\right) \mathrm{e}^{i \nu_{01}}$ is denoted $r_{m+1}^{\prime}$. Since we have $\left(\Psi_{\eta_{3}, \nu_{0},}\right.$ is orientation preserving):

$$
\Psi_{\eta_{3}, \nu_{0}}\left(\operatorname{arc}^{(R)}(\Lambda)\right) \subset \operatorname{arc}\left(\eta_{\left.\eta_{3}, \nu_{0}\right)}^{(R)} \subset \Psi_{\eta_{3}, \nu_{0}}\left(\operatorname{arc}^{(R)}\left(\Lambda^{\prime}\right)\right),\right.
$$

it follows that the vertices in $\partial r_{m+1}^{\prime}$ are just the $\bar{G}_{\left(\eta_{3}, \nu_{0}\right)}^{o}$-vertices which correspond (under $\Psi_{\eta_{3}, \nu_{0}}{ }^{\circ} \psi$ ) with the vertices in $\partial r_{m+1}$.

By induction assumption and in view of $\left(12^{\prime}\right)$ we have $\bar{G}^{\circ} \sim \bar{G}_{\left(\eta_{3}, \nu_{0}\right)}^{\circ}$ and the underlying graph isomorphism (say $\zeta$ ) is induced by $\Psi_{\eta_{3}, \nu_{0}} \circ \psi$.

Obviously, we can extend this equivalence to an equivalence between $\bar{G}$ and $\bar{G}\left(g_{\eta_{3}, \nu_{0}}\right)$ by defining $\zeta(x):=\gamma_{-}\left(\sigma_{\eta_{3}, \nu_{0}}\right)$; cf. Remark 3.3.

The angle of $\tau_{m+1}^{\prime}$ corresponding with the $\tau_{m+1}$-angle $\phi_{l}$ is denoted by $\phi_{l}^{\prime}$, $l=1, \ldots, s$. For $l=2,3, \ldots, s$ we have:

$$
\left|\phi_{l}-\phi_{i}^{\prime}\right|<\frac{\epsilon}{3 s} \text {. }
$$

This is a consequence of the induction assumption, $\left(12^{\prime}\right)$ and $\left(13^{\prime}\right)$. Since $\bar{G}$ as well as $\bar{G}\left(g_{\eta_{3}, \nu_{0}}\right)$ are Newton graphs, it follows, in view of $(20)$, that

$$
\left|\phi_{1}-\phi_{1}^{\prime}\right|=\left|\sum_{l=2}^{s} \phi_{l}-\phi_{l}^{\prime}\right|<\epsilon .
$$

This completes the proof of Step 2 and thus the proof of the theorem.

We conclude this section by deriving (separately) some results which we have already used in the proof of Theorem 4.1.

As usual, we denote by $D_{R}$ the disc $\{z|| z \mid \leqslant R\}$ in the $z$-chart of $S^{2}$, whereas $D_{R}^{w}$ stands for the disc $\{w \| w \mid \leqslant 1 / R\}$ in the $w$-chart of $S^{2} ; w=1 / z, R>0$. Let $f$ be a function in $\mathscr{R}$ (not necessarily in $\mathscr{R}_{+}$) and define $g_{\eta, \nu}$ as follows:

$$
g_{\eta, \nu}(z)=\left(-\eta \mathrm{e}^{-i \nu} z+1\right) \cdot f(z) \quad \eta, \nu \in \mathbf{R} .
$$


Then we have:

LEMMA 4.4. For any fixed, positive $R$ :

(i) $\lim _{\eta \downarrow}\left\|\left(\overline{\mathcal{N}}(f)-\overline{\mathcal{N}}\left(g_{\eta, \nu}\right)\right) \mid D_{\mathrm{R}}\right\|=0$, uniformly in $\nu$.

(ii) $\lim _{\nu \rightarrow \nu_{0}}\left\|\left(\overline{\overline{\mathcal{N}}}\left(g_{\eta_{1}, \nu}\right)-\overline{\overline{\mathcal{N}}}\left(g_{\eta_{0}, \nu_{0}}\right)\right) \mid D_{R}^{W}\right\|=0$, for each fixed pair $\left(\eta_{0}, \nu_{0}\right)$.

Here \|| || stands for the $C^{1}$-norm on the set of $C^{1}$-vector fields defined on $D_{R}$, respectively $D_{R}^{w}$.

Proof (Compare the formulae proceeding Lemmata 2.2 and $2.3 ; f=p_{n} / q_{m}$ ).

(i) One easily verifies that:

$$
\overline{\mathcal{N}}(f)=-\frac{\left|q_{m}(z)\right|^{2} \cdot p_{n}(z) \cdot \overline{p_{n}^{\prime}(z)}-\left|p_{n}(z)\right|^{2} \cdot q_{m}(z) \cdot \overline{q_{m}^{\prime}(z)}}{\left|q_{m}(z)\right|^{4}+\left|p_{n}(z)\right|^{4}}
$$

and

$$
\overline{\mathcal{N}}\left(g_{\eta, \nu}\right)=-\frac{\left|q_{m}(z)\right|^{2} \cdot p_{n}(z) \cdot \overline{p_{n}^{\prime}(z)}-\left|p_{n}(z)\right|^{2} \cdot q_{m}(z) \cdot \overline{q_{m}^{\prime}(z)}+\eta \cdot F_{1}(z, \bar{z}, \eta, \nu)}{\left|q_{m}(z)\right|^{4}+\left|-\eta \mathrm{e}^{-i \nu} z+1\right|^{4} \cdot\left|p_{n}(z)\right|^{4}},
$$

where $F_{1}$ stands for a polynomial in $z, \vec{z}, \eta, \mathrm{e}^{i \nu}$ and $\mathrm{e}^{-i \nu}$.

Consequently, we have:

$$
\overline{\mathcal{N}}(f)-\overline{\mathcal{N}}\left(g_{\eta, \nu}\right)=-\eta \frac{F_{2}(z, \bar{z}, \eta, \nu)}{\left(\left|q_{m}(z)\right|^{4}+\left|p_{n}(z)\right|^{4}\right) \cdot\left(\left|q_{m}(z)\right|^{4}+\left|-\eta \mathrm{e}^{-i \nu} z+1\right|^{4} \cdot\left|p_{n}(z)\right|^{4}\right)},
$$

where $F_{2}$ stands for a polynomial in $z, \bar{z}, \eta, \mathrm{e}^{i \nu}$ and $\mathrm{e}^{-i \nu}$.

Note that the denominator of the r.h.s. of the last expression is a polynomial in $z, \bar{z}, \eta$ and $\mathrm{e}^{ \pm i v}$ which - for $z \in D_{\mathbf{R}}$ and $\eta \in\left[0, \eta_{1}\right], \eta_{1}>0$ sufficiently small does not vanish. (Use the fact that $p_{n}, q_{m}$ are relatively prime.)

It follows that:

$$
\lim _{\eta \downarrow 0} \overline{\mathcal{N}}(f)-\overline{\mathcal{N}}\left(g_{\eta, \nu}\right) \mid D_{R}=0 .
$$

Since the expression for $\overline{\mathcal{N}}(f)-\overline{\mathcal{N}}\left(g_{\eta, \nu}\right)$ is a rational function in $z$ and $\bar{z}$, the components of this vector field are rational functions in $x$ and $y(x=\operatorname{Re} z$, $y=\operatorname{Im} z$ ), with denominators which do not vanish if $\eta$ is chosen sufficiently near to 0 .

The assertion follows immediately from this (the uniform convergence being a consequence of the fact that $\nu$ only appears in terms of the form $\mathrm{e}^{ \pm i \nu}$ ).

(ii) The proof follows by inspection of the expression for the vector field $\overline{\overline{\mathcal{N}}}\left(g_{\eta, \nu}\right)$.

Note that we must distinguish between the cases $n \neq m, n=m$; compare Lemma 2.3. Since the proof runs along the same lines as the proof of (i), we omit the tedious details.

COROLLARY 4.3. Let $\hat{g}_{\eta, \nu}$ be of the form $\hat{g}_{\eta, \nu}(z)=\left(-\eta \mathrm{e}^{-i \nu} z+1\right)^{-1} \cdot f(z)$. 
Then we have:

(i) $\lim _{\eta \downarrow}\left\|\left(\overline{\mathcal{N}}(f)-\overline{\mathcal{N}}\left(\hat{\mathrm{g}}_{\eta, \nu}\right)\right) \mid D_{R}\right\|=0$, uniformly in $\nu$.

(ii) $\left.\lim _{\nu \rightarrow \nu_{0}} \| \overline{\overline{\mathcal{N}}}\left(\hat{\mathrm{g}}_{\eta_{0}, \nu}\right)-\overline{\overline{\mathcal{N}}}\left(\hat{\mathrm{g}}_{\eta_{0}, \nu_{0}}\right)\right) \mid D_{R}^{w} \|=0$, for each fixed pair $\left(\eta_{0}, \nu_{0}\right)$.

Proof. This follows directly from the preceding lemma and the relations

$$
\overline{\mathcal{N}}(f)=-\overline{\mathcal{N}}\left(\frac{1}{f}\right), \quad \overline{\mathcal{N}}(\hat{\mathrm{g}})=-\overline{\mathcal{N}}\left(\frac{1}{\hat{\mathrm{g}}}\right), \quad \overline{\overline{\mathcal{N}}}(\hat{\mathrm{g}})=-\overline{\overline{\mathcal{N}}}\left(\frac{1}{\hat{\mathrm{g}}}\right)
$$

interchange the roles of $p_{n}$ and $q_{m}$.

LEMMA 4.5. The mappings $\chi_{1}, \chi_{2}$ (defined in the proof of Theorem 4.1) are continuous and surjective.

Proof. We only give the proof for $\chi_{1}$ since the proof for $\chi_{2}$ is similar. We emphasize that (see the proof of Theorem 4.1, Step 1) the numbers $R$ and $\eta$ are chosen in such a way that for all $\nu$ :

(1) $C_{R}$ is global boundary for $\overline{\mathcal{N}}\left(q_{\eta, v}\right)$.

(2) The functions $q_{\eta, \nu}$ have only one zero and one critical point (both simple) on $D_{R}^{w}$.

Moreover (see Lemma 2.4 and use: $q_{\eta, \nu} \in \mathscr{R}_{+}$), the systems $\overline{\overline{\mathcal{N}}}\left(q_{\eta, \nu}\right)$ have at $z=\infty$ a nondegenerate (unstable) node. We conclude that for each $\nu$, the system $\overline{\overline{\mathcal{N}}}\left(q_{\eta, \nu}\right) \mid D_{R}^{w}$ is $\epsilon$-structurally stable. In view of Lemma 4.4 (ii) and the definition of $\epsilon$-structural stability, one immediately derives the continuity of $\chi_{1}$.

Suppose $\chi_{1}$ is not surjective, i.e., $\hat{z} \in C_{R}$ exists such that $\hat{z} \notin \operatorname{Im} \chi_{1}$. Let $(1 / \eta) \mathrm{e}^{i \nu_{0}}$ be an arbitrary, but fixed point on $\{z|| z \mid=1 / \eta\}$.

The closed oriented continuous curve $\gamma_{\nu}$ is defined - for each $\nu, \nu \neq \nu_{0}(\bmod$ $2 \pi)$ - as the union of:

- $\left(\gamma_{-}\left(\sigma_{\eta, \nu_{1}}\right)\right) \cap\{z|| z \mid \geqslant R\}$ oriented from $(1 / \eta) \mathrm{e}^{i \nu_{0}}$ to $z_{\eta, \nu_{0}}^{*}$

- the arc of $C_{R}$ from $z_{\eta, \nu_{0}}^{*}$ to $z_{\eta, \nu}^{*}$ which does not contain $\hat{z}$;

$-\left(\gamma_{-}\left(\sigma_{\eta, \nu}\right)\right) \cap\{z|| z \mid \geqslant R\}$ oriented from $z_{\eta, \nu}^{*}$ to $(1 / \eta) \mathrm{e}^{i \nu}$;

- the $\operatorname{arc}$ of $\{z|| z \mid=1 / \eta\}$ from $(1 / \eta) \mathrm{e}^{i \nu}$ to $(1 / \eta) \mathrm{e}^{i \nu_{0}}$ with orientation in accordance with the anticlockwise orientation of $\{z|| z \mid=1 / \eta\}$.

Note that $\gamma_{\nu}$ is well-defined in view of the fact that $\hat{z} \notin \operatorname{Im} \chi_{1}$. See Figure 18 . The winding number $\omega_{0}\left(\gamma_{\nu}\right)$ of $\gamma_{\nu}$ around $z=0$ is well-defined:

$$
\omega_{0}\left(\gamma_{\nu}\right)=\frac{1}{2 \pi i} \oint_{\gamma_{\nu}} \frac{\mathrm{d} z}{z}
$$

In view of the $\epsilon$-structural stability of the system: $\overline{\overline{\mathcal{N}}}\left(q_{\eta, \nu}\right) \mid D_{R}^{w}$ (and the fact that $C_{R}$ is global boundary for $\overline{\overline{\mathcal{N}}}\left(q_{\eta, \nu}\right)$ ), it follows that $\omega_{0}\left(\gamma_{\nu}\right)$ depends continuously on $\nu$ (see [4]). Since $\omega_{0}\left(\gamma_{\nu}\right)$ is an integer we conclude, using the connectedness of

$$
\left\{z|| z \mid=\frac{1}{\eta}\right\} \backslash\left\{\frac{1}{\eta} \mathrm{e}^{i \nu_{0}}\right\}
$$




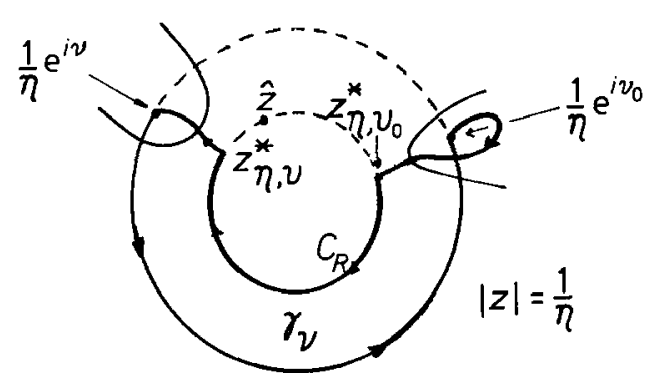

Fig. 18.

that $\omega_{0}\left(\gamma_{v}\right)=$ constant. But this is in contradiction with the fact (use again the $\epsilon$-structural stability of $\left.\overline{\overline{\mathcal{N}}}\left(q_{\eta, \nu_{0}}\right) \mid D_{R}^{w}\right)$ that

$$
\lim _{\nu \downarrow \nu_{0}} \omega_{0}\left(\gamma_{\nu}\right)-\lim _{\nu \uparrow \nu_{0}} \omega_{0}\left(\gamma_{\nu}\right)=\frac{1}{2 \pi i} \oint_{|z|=1 / \eta} \frac{\mathrm{d} z}{z}=1 \text {. }
$$

\section{The Classification Theorem and Some of Its Consequences}

In this section we classify the systems $\mathcal{N}(f)$, with $f \in \tilde{\mathscr{R}}_{+}$, in terms of Newton graphs. This classification involves a classification of all structurally stable rational Newton flows.

Let us consider a function $f \in \mathscr{R}$, such that $\mathcal{N}(f)$ is not a north-south flow. The (directed) sphere-graph $\overline{\bar{K}}(f)$, i.e. a realization of an abstract graph-say $K(f)$ in the sphere $S^{2}$, is defined as follows:

- The vertices of $\overline{\bar{K}}(f)$ are equilibrium-states of $\overline{\overline{\mathcal{N}}}(f)$.

- The edges of $\overline{\bar{K}}(f)$ are the closures of the separatrices at the saddlepoints of $\overline{\overline{\mathcal{N}}}(f)$ (the orientation being induced by the orientation of the trajectories of $\overline{\overline{\mathcal{N}}}(f))$.

Note that $\overline{\bar{H}}(f)$ and $\overline{\bar{H}}(1 / f)$ are connected subgraphs of $\overline{\bar{K}}(f)$, compare Remark 3.4. Consequently, the sphere graph $\overline{\bar{K}}(f)$ is connected.

Given a labeling of the edges of the abstract graph $K(f)$, underlying $\overline{\bar{K}}(f)$, the regions of $\overline{\bar{K}}(f)$ may be described by certain subsets of the set of labels. Following Peixoto [30], we call these subsets distinguished sets of $K(f)$. The graph $K(f)$, together with its distinguished sets is called the distinguished graph $K^{d}(f)$.

Two distinguished graphs are called isomorphic if they are isomorphic as abstract graphs and the isomorphism preserves the distinguished sets.

The following result is a direct consequence of Peixoto's classification (cf. [28], [30]).

LEMMA 5.1. Two structurally stable Newton flows on the sphere $S^{2}$ are equivalent 
iff either both the systems are north-south flows or the corresponding distinguished graphs are isomorphic.

The systems $\mathcal{N}\left(f_{1}\right)$ and $\mathcal{N}\left(f_{2}\right)$ are called topological equivalent $(\sim)$ if a homeomorphism $\mathbb{C} \rightarrow \mathbb{C}$ exists, which maps the trajectories of $\mathcal{N}\left(f_{1}\right)$ onto those of $\mathcal{N}\left(f_{2}\right)$ and preserves the orientations of the trajectories; compare the 'topological equivalence on $\chi\left(S^{2}\right)$ ' defined in Section 3 .

LEMMA 5.2. Let $f_{1}, f_{2} \in \tilde{R}_{+}$be given. Then $\mathcal{N}\left(f_{1}\right) \sim \mathcal{N}\left(f_{2}\right)$ iff $\bar{G}\left(f_{1}\right) \sim \bar{G}\left(f_{2}\right)$.

Proof. If at least one of the systems $\mathcal{N}\left(f_{1}\right), \mathcal{N}\left(f_{2}\right)$ is a north-south flow, then the theorem is obvious. Hence, we may assume that neither $\mathcal{N}\left(f_{1}\right)$ nor $\mathcal{N}\left(f_{2}\right)$ is a north-south flow.

As a direct consequence of the definitions of (topological) equivalence between Newton systems in the plane and between plane graphs (cf. Section 3), we have $\mathcal{N}\left(f_{1}\right) \sim \mathcal{N}\left(f_{2}\right)$ implies $\bar{G}\left(f_{1}\right) \sim \bar{G}\left(f_{2}\right)$. Now, suppose that $\bar{G}\left(f_{1}\right) \sim \bar{G}\left(f_{2}\right)$ with the underlying homeomorphism being denoted by $\psi$. Let $\pi$ be the sterographic projection from $\mathbb{C}$ onto $S^{2} \backslash\{z=\infty\}$ which maps the phase portraits of $\overline{\mathcal{N}}\left(f_{i}\right)$ onto those of $\overline{\overline{\mathcal{N}}}\left(f_{i}\right)$, cf. Lemma 2.3. Then, $\pi \circ \psi \circ \pi^{-1}$ can be extended to a homeomorphism $\psi^{*}$ from $S^{2}$ to $S^{2}$ which induces an equivalence between $\overline{\bar{G}}\left(f_{1}\right)$ and $\overline{\bar{G}}\left(f_{2}\right)$, cf. remark 3.4 .

A careful analysis (cf. [33]) of the properties of geometrical duals of sphere graphs, $\psi^{*}$ and $\overline{\bar{N}}\left(f_{i}\right)$, yields that $\psi^{*}$ induces an isomorphism between $K^{d}\left(f_{1}\right)$ and $K^{d}\left(f_{2}\right)$. Applying Lemma 5.1, we find that $\overline{\overline{\mathcal{N}}}\left(f_{1}\right) \sim \overline{\overline{\mathcal{N}}}\left(f_{2}\right)$, the equivalence being induced by a homeomorphism $\phi: S^{2} \rightarrow S^{2}$. Finally, the mapping $\pi^{-1} \circ \phi \circ \pi$ induces a homeomorphism between $\mathcal{N}\left(f_{1}\right)$ and $\mathcal{N}\left(f_{2}\right)$.

THEOREM 5.1 (Classification Theorem). Let $\bar{G}$ be a plane graph which either consists of only one vertex or fulfils the cycle property. Then, there exists a rational Newton flow $\mathcal{N}(f)$ with $f \in \mathscr{R}_{+}$, such that $\bar{G}(f) \sim \bar{G}$. This correspondence is bijective up to equivalence between rational Newton-flows and plane-graphs.

Proof. This follows directly from Remark 4.1, Corollary 4.2 and the Lemmata 4.2 and 5.2 .

In the case of functions $f \in \tilde{\mathscr{R}}_{-}$, the above theorem also provides a characterization of the systems $\mathcal{N}(f)$. This follows from the relation $\mathcal{N}(f)=-\mathcal{N}(1 / f)$.

In the case of functions $f \in \tilde{R}_{0}$, formal 'substitution' of $f$ (resp. 1/f) in Definition 3.1 does not generally yield a plane graph. This is due to the fact that $z=\infty$ may lie on an unstable manifold of a saddlepoint for $\overline{\overline{\mathcal{N}}}(f)\left(\operatorname{resp}_{.} \overline{\overline{\mathcal{N}}}(1 / f)\right)$. Moreover, if $z=\infty$ is a critical point for $f$, the saddlepoint $z=\infty$ for $\overline{\overline{\mathcal{N}}}(f)$ can 'move' into a finite saddlepoint for $\overline{\overline{\mathcal{N}}}(\tilde{f})$, where $\tilde{f}$ is obtained from $f$ by an arbitrary small perturbation of the coefficients of $f$. It follows that, although $\overline{\overline{\mathcal{N}}}(f)$ is structurally stable on the sphere, a similar regularity property is not necessarily true for the system $\overline{\mathcal{N}}(f)$ on the plane. This is a reflection of the fact that the 'natural way' of looking at rational Newton flows is to look at their spherical phase portraits (not 
the planar ones). Compare also Remark 5.1 below. So, let us consider $\overline{\overline{\mathcal{N}}}(f)$. Since $f \in \tilde{R}_{0}$, there is at least one zero, say $\omega$, and one pole, say $\alpha$. Under the transformation

$$
w=\frac{1}{z-\omega} \operatorname{resp} . w=\frac{1}{z-\alpha},
$$

the system $\overline{\overline{\mathcal{N}}}(f)$ changes over to the system $\overline{\overline{\mathcal{N}}}(g)$ resp. $\overline{\overline{\mathcal{N}}}(h)$ where $g$ (resp. $h)$ is of the form

$$
\frac{p_{n-1}}{q_{n}} \in \tilde{\mathscr{R}}_{-}\left(\text {resp. } \frac{p_{n}}{q_{n-1}} \in \tilde{\mathscr{R}}_{+}\right) .
$$

Thus, $\overline{\overline{\mathcal{N}}}(f)$ is equivalent with both $\overline{\overline{\mathcal{N}}}(g)$ and $\overline{\overline{\mathcal{N}}}(h)$, the latter systems - in their planar form - already being classified.

REMARK 5.1. Although, in Theorem 5.1 the very marrow of the classification of structurally stable rational Newton flows is presented, a more sophisticated approach by means of the sphere graphs $\overline{\bar{G}}(f)$ is possible (cf. [33]). In this context, systems $\overline{\overline{\mathcal{N}}}(f)$ with $f \in \tilde{\mathscr{R}}_{+}$are classified by sphere graphs $\overline{\bar{G}}$ which exhibits a region $r$ such that the stereographic projection, w.r.t. a point in $r$, transforms $\bar{G}$ onto a plane graph which fulfils the cycle property. We call these graphs $\overline{\bar{G}}$ admissible (w.r.t. the region $r$ ). The geometrical duals of such admissible graphs characterize the systems $\overline{\overline{\mathcal{N}}}(f)$ with $f \in \tilde{\mathscr{R}}_{-}$. The systems $\overline{\overline{\mathcal{N}}}(f)$ with $f \in \mathscr{R}_{0}$ are characterized by those admissible sphere graphs for which the geometrical dual is also admissible (balanced graphs). One easily sees (cf. [33]) that a sphere graph is balanced iff it is admissible w.r.t. each of its regions and, moreover, the number of its regions equals the number of its vertices.

By $\Sigma^{0}\left(S^{2}\right)$ we denote the subset of all structurally stable systems in $\chi\left(S^{2}\right)$ which do not exhibit periodic trajectories. For a system $X \in \Sigma^{0}\left(S^{2}\right)$, a sphere graph $\overline{\bar{G}}(X)$ and a distinguished graph $K^{d}(X)$ can be introduced in the same way as we defined $\overline{\bar{G}}(f)$ and $K^{d}(f)$ for $f \in \tilde{R}$. By means of these graphs, Peixoto [30] has given a complete characterization of the systems in $\Sigma^{0}\left(S^{2}\right)$. Using this result as well as our characterization theorem, one easily shows that any system $X \in \Sigma^{0}\left(S^{2}\right)$ which has only one unstable and $n$ stable nodes, is equivalent with a Newton system $\overline{\overline{\mathcal{N}}}\left(p_{n}\right)$, where $p_{n}$ is a polynomial in $\tilde{\mathscr{R}}$ of degree $n$. For details and another result in the same spirit we refer to [33].

Harary et al. (cf. [9-11]) have counted the number of various kinds of plane graphs (especially plane trees) up to orientation-preserving plane homeomorphisms. In view of Theorem 5.1, it is therefore reasonable to ask for the number $(\# \mathcal{N}(f))$ of systems $\mathcal{N}(f), f \in \tilde{R}$, up to topological equivalence. It turns out that in the special cases where $p_{n}$ is a polynomial in $\tilde{R}_{+}$of degree $n$ and where $f_{n}(\in \mathscr{R})$ is of the form

$$
f_{n}(z)=\frac{p_{n}(z)}{z-z_{0}}, \quad \text { with } p_{n}\left(z_{0}\right) \neq 0,
$$


Table I

\begin{tabular}{lrrrrrrrrrrrr}
\hline$n$ & 1 & 2 & 3 & 4 & 5 & 6 & 7 & 8 & 9 & 10 & 11 & $\cdots$ \\
\hline$\# \mathcal{N}\left(p_{n}\right)$ & 1 & 1 & 1 & 2 & 3 & 6 & 12 & 27 & 65 & 175 & 490 & $\cdots$ \\
\hline$\# \mathcal{N}\left(f_{n}\right)$ & 1 & 1 & 2 & 5 & 11 & 30 & 79 & 237 & 730 & 2388 & 8021 & $\cdots$ \\
\hline
\end{tabular}

we have:

$$
\# \mathcal{N}\left(p_{n}\right)=\frac{1}{2}\left(\left(a_{n}+b_{n}\right) \text { and } \# \mathcal{N}\left(f_{n}\right)=\frac{1}{2}\left(g_{n+1}+t_{n+1}-f_{n+1}-p_{n+1}\right),\right.
$$

where $a_{n}, q_{n}, \iota_{n}, f_{n}$ and $p_{n}$ stand for, respectively, the number of achiral-, rooted-, achiral rooted-, planted- and achiral planted-, plane trees on $n$ vertices, and where $b_{n}$ is the number of the plane trees on $n$ vertices (all counted up to orientation-preserving plane homeomorphisms). Note that complications arise, since Harary's concept of equivalence between plane graphs is more restrictive (orientation is preserved) than our concept. Here, we merely present our results, cf. Table I.

\section{Asymptotic Values for Meromorphic Functions}

There is a strong relationship between (some aspects) of the theory on asymptotic values of meromorphic functions and the theory on Newton flows (cf. Section 2). This is not surprising since the trajectories of $\mathcal{N}(f)$ are contained in the lines $\arg f(z)=$ const. and, moreover, on a trajectory, $|f(z)|$ is strictly monotone (cf. Relation (2.1)). In this section we merely state our results; for the proofs we refer to [19].

Let $f$ be a meromorphic function. An asymptotic path for $f$, with asymptotic value $\alpha$ is a continuous curve $\gamma$, tending to $z=\infty$, and such that if $z \rightarrow \infty$ along $\gamma$, then $f(z) \rightarrow \boldsymbol{\alpha}$. (Here, $\alpha=\infty$ is possible). An asymptotic path for $f$ which is also a trajectory for $\mathcal{N}(f)$ is called a Newton asymptotic path.

We begin by presenting a result which - for a simple class of entire functions constitutes a refinement of the classical theorem of Denjoy-Ahlfors and of Julia $[24,33]$ and which is an application of Lemma 2.4b. To this aim, we introduce the concept of Julia-line:

Let $g$ be an entire function. A ray $\rho$, emanating from the origin $z=0$ is called a Julia-line for $g$ if: given any $z \in \mathbf{C}$ (with eventually one exception) and any angle with $\rho$ as its bisector, $g$ takes the value $z$ at every point of an infinite sequence, converging to $z=\infty$ and lying within this angle.

THEOREM 6.1. Let $g$ be an entire function of order $l<\infty$ and with $\alpha(\in \mathbb{C})$ as its Picard exceptional value, i.e., $(g-\alpha) \in \mathscr{E}$; cf. [15]. Then 
(1) There are exactly $2 l$ Julia-lines for $g$; two adjacent lines intersect under equal angles $\pi / l$.

(2) The only asymptotic values for $g$ are $\alpha$ and $\infty$.

We proceed by stating a result on Newton asymptotic paths for meromorphic functions.

THEOREM 6.2. Let $f$ be a nonconstant meromorphic function. Suppose that $z^{*} \in N(f)$ and $\partial B\left(z^{*}\right) \neq \emptyset$ (i.e., $f$ is not of the form $a\left(z-z^{*}\right)^{n}, a \neq 0$; compare Section 2). Then, at least one of the following properties holds:

(1) There is at least one (finite) critical point on the boundary $\partial B\left(z^{*}\right)$.

(2) There exists a Newton asymptotic path for $f-\operatorname{say} \gamma\left(z_{0}\right)-$ with $z_{0} \in B\left(z^{*}\right)$ which has a finite, nonzero asymptotic value.

Using Theorems 6.1 and 6.2 we derived the following result in [19].

THEOREM 6.3. Let $\mathrm{g}$ be as in Theorem 6.1 and $\alpha \neq 0$ (thus $\mathrm{g}$ has infinitely many zeros). Then, with the eventual exception of a finite number, for all zeros $z^{*}$, there are at least one and at most $k$ Newton asymptotic paths for $\mathrm{g}$, issuing from $z^{*}$ with asymptotic value $\alpha$. Here $k$ stands for the multiplicity of $z^{*}$ (as a zero of $g$ ).

\section{Acknowledgement}

We want to thank Heinz-Otto Peitgen for encouraging us to write this paper.

Added in proof. In the special case of plane trees, an independent proof of the Representation Theorem (Corollary 4.2) is given by M. Shub and B. Williams, The Newton graph of a complex polynomial, Preprint (1985), and by ourselves [19]. See also D. Braess [2] and S. Smale, On the efficiency of algorithms in analysis, Bull. Am. Math. Soc. 13-2 (1985), 87-121, who posed this result as an open problem.

\section{References}

1. Andronov, A. A., Leontovich, E. L., Gordon, I. I., and Maier, A. G.: Theory of bifurcations of dynamical systems on a plane, Wiley, New York, 1973.

2. Braess, D.: Ueber die Einzugbereiche der Nullstellen von Polynomen beim Newton-Verfahren, Numer. Math. 29 (1977), 123-132.

3. Branin, F. H.: A widely convergent method for finding multiple solutions of simultaneous non-linear equations, I.B.M. J. Res. Develop. (1972), 504-522.

4. Deimling, K.: Nicht-linearen Gleichungen und Abildungsgrade, Hochschultext, Springer-Verlag, Berlin, 1974.

5. Garcia, C. B. and Gould, F. J.: Relations beteween several path-following algorithms and local and global Newton methods, SIAM Rev., 22, (1980) 263-274.

6. Giblin, P. J.: Graphs, Surfaces and Homology, Wiley, New York, 1977.

7. Gomulka, J.: Remarks on Branin's method for solving non-linear equations, in L. C. W. Dixon and G. P. Szegö (eds.), Towards Global Optimization Academic Press, New York, 1976. 
8. Guillemin, V. and Pollack, A.: Differential Topology, Prentice Hall, Englewood Cliffs, 1974.

9. Harary, F., Prins, G., and Tutte, W. T.: The number of plane trees, Indag. Math. 26 (1964), 319-329.

10. Harary, F.: Graph Theory, Addison-Wesley, Reading, Mass., 1969.

11. Harary, F. and Robinson, R. W.: The number of achiral trees, J. Reine Angew. Math. 278 (1975), 322-335.

12. Hartman, P.: Ordinary Differential Equations, John Wiley, New York, 1964.

13. Hirsch, M. W. and Smale, S.: Differential Equations, Dynamical Systems and Linear Algebra, Academic Press, New York, 1970.

14. Hirsch, M. W. and Smale, S.: Algorithms for solving $f(x)=0$, Comm. Pure Appl. Math. 32 (1979), 281-312.

15. Holland, A. S. B.: Introduction to the Theory of Entire Functions, Academic Press, New York, 1973.

16. Hurewicz, W.: Lectures on Ordinary Differential Equations, MIT Press, Cambridge, Mass., 1970.

17. Hurley, M. and Martin, C.: Newton's algorithm and chaotic dynamical systems, SIAM J. Math. Anal. 15 (1984), 238-252.

18. Jongen, H. Th., Jonker, P., and Twilt, F.: On Newton-flows in optimization, Methods of Operations Research 31 (1979), 345-359.

19. Jongen, H. Th., Jonker, P., and Twilt, F.: The continuous Newton method for meromorphic functions, in R. Martini, (ed.), Geometric Approaches to Differential Equations, Lect. Notes in Math., Vol. 810, Springer-Verlag, Berlin 1980, pp. 181-239.

20. Jongen, H. Th., Jonker, P., and Twilt, F.: Some reflections on the continuous Newton-method for rational functions, in L. Collatz, G. Meinardus and W. Wetterling, (eds.), Konstruktive Methoden der finiten nichtlinearen Optimierung Int. Series Num. math., Vol. 55, 1980, pp. 131-147.

21. Jongen, H. Th., Jonker, P., and Twilt, F.: On the classification of plane-graphs representing structurally stable rational Newton flows, Memorandum $\mathrm{Nr} 645$, University of Twente, Enschede. The Netherlands (1987).

22. Lefschetz, S.: Differential Equations: Geometric Theory, Interscience Publ.

23. Markushevich, A. I.: Theory of Functions of a Complex Variable, Vol. II, Prentice Hall, Englewood Cliffs, 1965.

24. Markushevich, A. I.: Theory of Functions of a Complex Variable, Vol. III, Prentice Hall, Englewood Cliffs, 1965.

25. Milnor, J. W.: Topology from a Differential Viewpoint, The University Press of Virginia, 1965.

26. Nevanlinna, R.: Eindeutige Analytische Funktionen, Springer-Verlag, Berlin, 1957.

27. Peitgen, H. O., Saupe, D., and Haeseler, F. V.: Newton's methods and Julia sets, Report No. 104, Forschungsschwerpunkt Dynamische Systeme, Universität Bremen (1983).

28. Peixoto, M. C. and Peixoto, M. M.: Structural stability in the plane with enlarged boundary conditions, An. Acad. Bras. de Ciêns, (1959), 135-160.

29. Peixoto, M. M.: Structural stability on two-dimensional manifolds, Topology 1 (1962), 101-120.

30. Peixoto, M. M.: On the classification of flows on 2-manifolds, in M. M. Peixoto, (ed.), Dynamical Systems. Academic Press, New York, 1973, pp. 389-419.

31. Smale, S.: A convergent process of price adjustment and global Newton-methods, J. Math. Economics 3 (1976), 107-120.

32. Smale, S.: The fundamental theorem of algebra and complexity theory, Bull. Am. Math. Soc., (1981), 1-36.

33. Twilt, F.: Newton-flows for meromorphic functions, Ph.D. Thesis, Twente University of Technology, The Netherlands, 1981. 